JOURNAL OF

SYMPLECTIC GEOMETRY

Volume 5, Number 4, 385-437, 2007

\title{
AN ALGEBRAIC FORMULATION OF SYMPLECTIC FIELD THEORY
}

\author{
ERIC KATZ
}

\begin{abstract}
We develop a formalism for relative Gromov-Witten invariants following Li J. Li, Stable morphisms to singular schemes and relative stable morphisms, J. Differential Geom. 57(3) (2001), 509-578, J. Li, A degeneration formula of $G W$-invariants, J. Differential Geom. 60(2) (2002), 199-293 that is analogous to the symplectic field theory (SFT) of Eliashberg, Givental and Hofer Y. Eliashberg, A. Givental and H. Hofer, Introduction to symplectic field theory, Geom. Funct. Anal.(Special Volume, Part II) (2000), 560-673 GAFA 2000 (Tel Aviv, 1999). This formalism allows us to express natural degeneration formulae in terms of generating functions and re-derive the formulae of Caporaso-Harris L. Caporaso and J. Harris, Counting plane curves of any genus, Invent. Math. 131(2) (1998), 345-392, Ran Z. Ran, Enumerative geometry of singular plane curves, Invent. Math. 97(3) (1989), 447-465, and Vakil $\mathrm{R}$. Vakil, The enumerative geometry of rational and elliptic curves in projective space, J. Reine Angew. Math. 529 (2000), 101-153 for counting rational curves. In addition, our framework gives a homology theory analogous to SFT homology.
\end{abstract}

\section{Introduction}

Relative Gromov-Witten invariants following Li $[\mathbf{1 5}, \mathbf{1 6}]$ and the symplectic field theory (SFT) of Eliashberg, Givental, and Hofer [2] are both theories of holomorphic curves with asymptotic boundary conditions. They have different sources: the theory of relative Gromov-Witten invariants counts stable maps to a projective manifold relative a divisor and is a systematization of degeneration methods in enumerative geometry $[\mathbf{1}, \mathbf{2 0}, \mathbf{2 2}]$; SFT is a generalization of Floer homology. SFT has an interesting formal structure involving a differential graded algebra whose homology is an invariant of contact structures. 
In relative Gromov-Witten theory, one considers a pair $(Z, D)$, where $Z$ is a projective manifold and $D$ is a smooth, possibly disconnected divisor in $Z$. One looks at stable maps to $Z$ where all points of intersections of the map with $D$ are marked and multiplicities at these points are specified. To obtain a proper moduli stack of such maps, one must allow the target to degenerate to ${ }_{k} Z=Z \sqcup_{D} P_{1} \sqcup_{D} \cdots \sqcup_{D} P_{k}$, that is, $Z$ union a number of copies of $P=\mathbb{P}_{D}\left(N_{D / Z} \oplus 1_{D}\right)$, the projective completion of the normal bundle to $D$ in $Z$. Maps with a non-smooth target are said to be split maps. Li defined a moduli stack of relative maps called $\mathcal{M}(\mathcal{Z}, \Gamma)$ for $\Gamma$, a topological type, and constructed its virtual fundamental cycle. This stack has an evaluation map

$$
\operatorname{Ev}_{\mathcal{M Z}}: \mathcal{M}(\mathcal{Z}, \Gamma) \longrightarrow Z^{m} \times D^{r}
$$

where $m$ and $r$ are the number of interior and boundary marked points, respectively. Relative Gromov-Witten invariants are given by evaluating pullbacks of cohomology classes by Ev against the virtual cycle.

It is natural to break the target ${ }_{k} Z$ as the union of

$$
{ }_{l} Z=Z \sqcup_{D} P_{1} \sqcup_{D} \cdots \sqcup_{D} P_{l}
$$

and

$$
{ }_{k-l-1} P=P_{l+1} \sqcup_{D} \cdots \sqcup_{D} P_{k} .
$$

In fact, such splitting is necessary to parameterize fixed loci in $\mathbb{C}^{*}$ localization in the sense of $[\mathbf{1 2}]$ and $[\mathbf{8}]$ in the relative framework $[\mathbf{9}]$. If we set $X=D$, and $L=N_{D / Z}$, the normal bundle to $D$ in $Z$, one is led to study stable maps into the projectivization of a line-bundle $P=\mathbb{P}_{X}\left(L \oplus 1_{X}\right)$ relative to its zero and infinity sections, $D_{0}$ and $D_{\infty}$ where two stable maps are declared equivalent if they can be related by a $\mathbb{C}^{*}$-factor dilating the fibers of $P \longrightarrow X$. One can construct a moduli stack of such maps, $\mathcal{M}(\mathcal{A}, \Gamma)$ and its virtual cycle. This moduli stack has certain natural line-bundles, called the target cotangent line-bundles, $\mathrm{L}^{0}$ and $\mathrm{L}^{\infty}$ and has an evaluation map

$$
\operatorname{Ev}_{\mathcal{M A}}: \mathcal{M}(\mathcal{A}, \Gamma) \longrightarrow X^{m} \times X^{r_{0}} \times X^{r_{\infty}} .
$$

The rubber invariants are obtained by evaluating pullbacks of cohomology by Ev and powers of $c_{1}\left(\mathrm{~L}^{\infty}\right)$ against the virtual cycle.

The purpose of this paper is a systematic development of the formal structure of relative Gromov-Witten invariants, organized in generating functions.

We note here that the rubber invariants have been introduced previously in the literature by Okounkov and Pandharipande [19] and by Graber and Vakil [9] as maps to a non-rigid target.

In Section 2, we recall the necessary background information to describe the stacks $\mathcal{M}\left(\mathcal{Z}, \Gamma_{Z}\right)$ and $\mathcal{M}\left(\mathcal{A}, \Gamma_{A}\right)$. We show how to glue together such 
stacks to parameterize split maps in a stack $\mathcal{M}\left(\mathcal{Z}, \Gamma_{Z} * \Gamma_{A}\right)$. We describe line-bundles on $\mathcal{M}(\mathcal{Z}, \Gamma)$ : Dil and $\mathrm{L}_{i \text {,ext }}$; and line-bundles on $\mathcal{M}(\mathcal{A}, \Gamma)$ : Split, $\mathrm{L}^{0}, \mathrm{~L}^{\infty}, \mathrm{L}_{i, \text { not top }}$, and $\mathrm{L}_{i \text {,not bot }}$. These line-bundles have geometric meaning: $\mathrm{L}_{i \text {,ext }}$ is a line-bundle which has a section whose zero-stack consists of maps $f: C \longrightarrow{ }_{k} Z$ so that the $i$ th marked point is not mapped to $Z \subset{ }_{k} Z$ (counted with multiplicity); Split is a line-bundle whose zero-stack is all split maps; $\mathrm{L}_{i \text {,not top }}$, where $i$ is the label of interior marked point, is a linebundle whose zero-stack consists of all split maps $f: C \longrightarrow{ }_{k} P$ where $i$ th

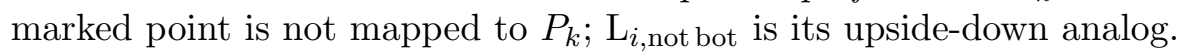

These line-bundles satisfy certain relations. On $\mathcal{M}\left(\mathcal{Z}, \Gamma_{Z}\right)$ :

$$
\mathrm{ev}_{i}^{*} \mathcal{O}(D)=\mathrm{L}_{i, \mathrm{ext}}
$$

and on $\mathcal{M}\left(\mathcal{A}, \Gamma_{A}\right)$ :

$$
\begin{aligned}
\mathrm{L}^{0} \otimes \mathrm{L}^{\infty} & =\text { Split } \\
\mathrm{L}^{0} \otimes \mathrm{ev}_{i}^{*} L^{\vee} & =\mathrm{L}_{i, \text { not top }} \\
\mathrm{L}^{\infty} \otimes \mathrm{ev}_{i}^{*} L & =\mathrm{L}_{i, \text { not bot }} .
\end{aligned}
$$

In Section 3, we organize intersection numbers on $\mathcal{M}(\mathcal{Z}, \Gamma)$ and $\mathcal{M}(\mathcal{A}, \Gamma)$ into generating functions. The intersection numbers on $\mathcal{M}(\mathcal{Z}, \Gamma)$ of the form

$$
\operatorname{deg}\left(\operatorname{Ev}_{\mathcal{M Z}}{ }^{*} c \cap[\mathcal{M}(\mathcal{Z}, \Gamma)]^{\operatorname{vir}}\right)
$$

are organized into the relative potential $F$ which takes values in a particular graded algebra $\mathcal{F}$. The intersection numbers on $\mathcal{M}(\mathcal{A}, \Gamma)$,

$$
\operatorname{deg}\left(c_{1}\left(\mathrm{~L}^{\infty}\right)^{l} \cup \operatorname{Ev}_{\mathcal{M A}}{ }^{*} c \cap[\mathcal{M}(\mathcal{A}, \Gamma)]^{\mathrm{vir}}\right)
$$

are organized into the rubber potential $A$ which takes values in an algebra $\mathcal{R}$. The algebra $\mathcal{R}$ acts on $\mathcal{F}$ which corresponds to joining curves in $\mathcal{M}\left(\mathcal{Z}, \Gamma_{Z}\right)$ and $\mathcal{M}\left(\mathcal{A}, \Gamma_{A}\right)$ to form split maps in $\mathcal{M}\left(\mathcal{Z}, \Gamma_{Z} * \Gamma_{A}\right)$. Likewise, the multiplication operation in $\mathcal{R}$ corresponds to joining curves in $\mathcal{M}\left(\mathcal{A}, \Gamma_{A b}\right)$ to those in $\mathcal{M}\left(\mathcal{A}, \Gamma_{A t}\right)$.

In Section 4, we prove degeneration formulae for the relative and rubber potentials. These degeneration formulae are differential equations satisfied by the potentials and are numerical consequences of the relations between line-bundles. Let $F$ be the relative potential of a pair $(Z, D)$ and let $A_{\lambda=0}$ be the rubber potential of the pair $\left(D, L=N_{D / Z}\right)$ without any powers of $c_{1}\left(\mathrm{~L}^{\infty}\right)$. Then, $F$ satisfies the differential equation

$$
\sum_{l} N_{j l} \frac{\partial F}{\partial \theta_{l}}=\sum_{l} M_{j l} \frac{\partial A_{\lambda=0}}{\partial \beta_{l}} \cdot F,
$$

where $\theta$ and $\beta$ are variables dual to cohomology classes on $Z$ and $X$, respectively, $M_{j l}$ and $N_{j l}$ are matrices that keep track of cohomology information, and · is the action of $\mathcal{R}$ on $\mathcal{F}$. 
Given a pair $(X, L)$, the rubber potential satisfies the analogous differential equation

$$
\frac{\partial}{\partial \lambda} \frac{\partial A}{\partial \beta_{i}}+\sum_{j} N_{i j} \frac{\partial A}{\partial \beta_{j}}=\frac{\partial A_{\lambda=0}}{\partial \beta_{i}} * A,
$$

where $*$ is multiplication in $\mathcal{R}$.

In Section 5, we work out several examples. We express the rational rubber potential without powers of $c_{1}\left(\mathrm{~L}^{\infty}\right)$ of $\left(\mathbb{P}^{n}, \mathcal{O}(m)\right)$ in terms of the GromovWitten invariants of $\mathbb{P}^{n}$ by a Kleiman-Bertini argument. We use this rubber potential to write down a degeneration formula for the relative GromovWitten potential of $\left(\mathbb{F}_{n}, D_{\infty}\right)$ and $\left(\mathbb{P}^{n}, H\right)$, where $D_{\infty} \subset \mathbb{F}_{n}$ is the infinity section of the rational ruled surface of degree $n$, and $H$ is a hyperplane in $\mathbb{P}^{n}$. This immediately yields the degeneration formulae of Caporaso-Harris [1], Ran [20], and Vakil [22], phrased in the language of differential operators as first stated by Getzler in [6].

In Section 6, we construct a theory directly analogous to SFT. One begins with a pair $(X, L)$ and organizes a subset of the rubber invariants into a generating function $H$ called the Hamiltonian that takes values in an algebra $\mathcal{H}$.

Given two interior marked points, one has the following formula among divisors on $\mathcal{M}(\mathcal{A}, \Gamma)$ :

$$
\mathrm{ev}_{2}^{*}\left(c_{1}(L)\right)-\mathrm{ev}_{1}^{*}\left(c_{1}(L)\right)=\underbrace{1 f}_{2}-{ }_{1}^{2 f}
$$

where the figures on the right specify certain loci of split maps. As a consequence of this formula, we have in $\mathcal{H}$,

$$
H^{2}=0 \text {. }
$$

We can then define a differential on $\mathcal{H}$ by the formula

$$
D^{H}=H f-(-1)^{\operatorname{deg} f} f H .
$$

The homology of this complex, called Hamiltonian homology, is an invariant of $(X, L)$ and is the algebraic geometric analog of the SFT homology of $S^{1}(L)$, the unit circle bundle of $L$.

In Section 7, we give a direct proof of the degeneration formula for the rubber potential using the technique of virtual localization.

This paper draws most directly on the relative Gromov-Witten invariants constructed by $\mathrm{Li}[\mathbf{1 5}, \mathbf{1 6}]$ and the SFT of Eliashberg, Givental, and Hofer [2]. Other approaches to relative invariants include those of Gathmann [4], Ionel and Parker [10], and Li and Ruan [14].

The author would like to acknowledge the following for valuable conversations: Eliashberg, Gathmann, Li, and Vakil. This paper, together with [11] 
make up a revised version of the author's Ph.D. thesis written under the direction of Eliashberg.

All varieties are over $\mathbb{C}$.

\section{Background}

We discuss stacks of relative stable maps, $\mathcal{M Z}=\mathcal{M}(\mathcal{Z}, \Gamma)$ and stacks of maps to rubber, $\mathcal{M A}=\mathcal{M}(\mathcal{A}, \Gamma)$, where $\Gamma$ specifies the topological type of the map. The material in this section is a rephrasing of sections of [11], some of which is a straightforward adaptation of [15] and [16]. While Li does not construct $\mathcal{M A}$, our construction directly parallels the construction of his moduli stacks. We do change some notation from [15] to suit our purposes.

2.1. Stacks of relative maps. Consider a projective manifold $Z$ with a smooth divisor $D$. We review the construction of the stack of stable maps to $Z$ relative to $D$. Given an $r$-tuple of positive integers $\mu=\left(\mu_{1}, \ldots, \mu_{r}\right)$, consider marked pre-stable curves

$$
\left(C, x_{1}, \ldots, x_{m}, p_{1}, \ldots, p_{r}\right)
$$

and maps

$$
f: C \longrightarrow Z
$$

so that the divisor $f^{*} D$ is

$$
f^{*} D=\sum_{i} \mu_{i} p_{i}
$$

To form a proper moduli stack of such maps, we must allow the target to degenerate. Let $L=N_{D / Z}$ be the normal bundle to $D$ in $Z$. Let $P=$ $\mathbb{P}_{D}\left(L \oplus 1_{D}\right)$ be the projective completion of $L$. $P$ has two distinguished divisors, $D_{0}$ and $D_{\infty}$, the zero and infinity sections of $L$.

Definition 2.1. Let ${ }_{k} Z$ be the union of $Z$ with $k$ copies of $P, Z \sqcup_{D} P_{1}$ $\sqcup_{D} \cdots \sqcup_{D} P_{k}$, the scheme given by identifying $D \subset Z$ with $D_{\infty} \subset P_{1}$ and $D_{0} \subset P_{i}$ with $D_{\infty} \subset P_{i+1}$ for $i=0,1, \ldots, k-1$.

Definition 2.2. Let $c:{ }_{k} Z \longrightarrow Z$ be the collapsing map that is the identity on $Z$ and projects each $P_{i}$ to $D \subset Z$.

Note that $\operatorname{Sing}\left({ }_{k} Z\right)$, the singular locus of ${ }_{k} Z$ is the disjoint union of $k-1$ copies of $D$, which we label $D_{1}, \ldots, D_{k-1}$ where $D_{i}=D_{\infty} \subset P_{i}$.

Definition 2.3. Let $\operatorname{Aut}\left({ }_{k} Z\right)=\left(\mathbb{C}^{*}\right)^{k}$ be the group acting on ${ }_{k} Z$ where each factor of $\mathbb{C}^{*}$ dilates the fibers of the $\mathbb{P}^{1}$ bundle $P_{i} \longrightarrow X$. As we will be considering maps to be equivalent if they are related by this action, the explicit form of this action does not matter.

Definition 2.4. Let $D \subset{ }_{k} Z$ denote the divisor $D_{0} \subset P_{k} \subset{ }_{k} Z$. 
We need to specify the appropriate data for the moduli stack of relative stable maps to $(Z, D)$. We consider an algebraic curve $C$ that is mapped to ${ }_{k} Z$ by $f: C \longrightarrow{ }_{k} Z$ with specified tangency to $D$. We must specify the topology of the curve and the data of the marked points. There are two types of marked points:

(1) interior marked points whose image under $f$ is not mapped to $D$;

(2) boundary marked points which are mapped to $D$ by $f$.

We will impose the condition that all points in $C$ mapped to $D$ will be marked.

Definition 2.5. A relative topological type $\Gamma$ is the following data:

(1) A finite set $V(\Gamma)$;

(2) A genus assignment

$$
g: V(\Gamma) \longrightarrow \mathbb{Z}_{\geq 0}
$$

(3) A degree assignment

$$
d: V(\Gamma) \longrightarrow B_{1}(Z) \equiv A_{1}(Z) /{ }^{\sim} \text { alg }
$$

that assigns the class of a curve modulo algebraic equivalence;

(4) A set $R=\{1, \ldots, r\}$ labeling boundary marked points together with a function assigning boundary marked points to elements of $V(\Gamma)$,

$$
a_{R}: R \longrightarrow V(\Gamma)
$$

(5) A multiplicity assignment for each boundary marked point

$$
\mu: R \longrightarrow \mathbb{Z}_{\geq 1}
$$

(6) A set $M=\{1, \ldots, m\}$ labeling interior marked points together with an assignment

$$
a_{M}: M \longrightarrow V(\Gamma) .
$$

Note that while the definition of $\Gamma$ is reminiscent of that of a graph, $\Gamma$ does not have any edges. We will occasionally use $R_{Z}$ and $M_{Z}$ to denote $R$ and $M$ where there is a possibility of confusion.

Definition 2.6. Two relative topological types $\Gamma, \Gamma^{\prime}$ are said to be isomorphic if there is a bijection

$$
q: V(\Gamma) \longrightarrow V\left(\Gamma^{\prime}\right)
$$

commuting with the maps $g, d, a_{R}, a_{M}$.

Definition 2.7. Let $\Gamma$ be a relative topological type. A map to ${ }_{k} Z$ of type $\Gamma$ consists of a marked curve $\left(C, x_{1}, \ldots, x_{|M|}, p_{1}, \ldots, p_{|R|}\right)$ and a morphism $f: C \longrightarrow{ }_{k} Z$ such that

(1) $C$ is a disjoint union of pre-stable curves $C_{v}$ for $v \in V(\Gamma)$,

(2) $C_{v}$ is a connected curve of arithmetic genus $g(v)$, 
(3) The map

$$
(c \circ f): C_{v} \longrightarrow{ }_{k} Z \longrightarrow Z
$$

has $(c \circ f)_{*} C_{v}=d(v) \in A_{1}(Z) \Gamma_{\mathrm{alg}}$,

(4) $x_{i} \in C_{v}$ for $v=a_{M}(i)$ which are called the interior marked points,

(5) $p_{i} \in C_{v}$ for $v=a_{R}(i)$ which are called the boundary marked points,

(6) $f^{*} D=\sum_{i \in R} \mu(i) p_{i}$.

Definition 2.8. A map $f: C \longrightarrow{ }_{k} Z$ is said to be pre-deformable if for $i=1, \ldots, k-1, f^{-1}\left(D_{i}\right)$ is the union of nodes so that for $p \in f^{-1}\left(D_{i}\right)$, the two branches of the node are mapped to different irreducible components of ${ }_{k} Z$ and that the order of contact to $D_{i}$ are equal.

Definition 2.9. An isomorphism between maps $f, f^{\prime}$ to ${ }_{k} Z$ is a commutative diagram

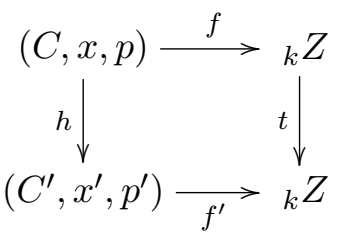

where $h$ is an isomorphism of marked curves and $t \in \operatorname{Aut}\left({ }_{k} Z\right)$.

Definition 2.10. A pre-deformable map to ${ }_{k} Z$ is said to be stable if it has at most finitely many automorphisms.

Theorem 2.11 ([15]). For a relative topological type $\Gamma$, there is a DeligneMumford stack, called the stack of relative stable maps, $\mathcal{M}(\mathcal{Z}, \Gamma)$, parameterizing isomorphism classes of stable pre-deformable maps to ${ }_{k} Z$ for varying $k$.

In cases where it is understood, we will write $\mathcal{M Z}$ for $\mathcal{M}(\mathcal{Z}, \Gamma)$

This moduli stack is constructed from a moduli functor by considering stable maps to targets modeled on a sequence of spaces and divisors $(Z[n], D[n])$ defined inductively by

$$
\begin{aligned}
& Z[0]=Z \\
& D[0]=D \\
& Z[n]=\operatorname{Bl}_{D[n-1] \times\{0\}}\left(Z[n-1] \times \mathbb{A}^{1}\right)
\end{aligned}
$$

and $D[n]$ is the proper transform of $D[n-1] \times \mathbb{A}^{1} . Z[n] / Z$ possesses a $\left(\mathbb{C}^{*}\right)^{n}$ group of automorphisms. This construction is an iteration of deformation to the normal cone. $Z[n]$ possesses a map to $\mathbb{A}^{n}$ so that for a closed point $x \in \mathbb{A}^{n}$, the fiber over $x$ is $(Z[n])_{x}={ }_{k} Z$, where $k$ is the number of zeroes among $x$ s coordinates.

Definition 2.12. A map $f: C \longrightarrow{ }_{k} Z$ is said to be split if $k \geq 1$. The irreducible components of $C$ that are mapped to $P_{i} \subset{ }_{k} Z$ are said to be extended components. 
Definition 2.13. The evaluation map on $\mathcal{M Z}$ is a map

$$
\mathrm{Ev}: \mathcal{M Z} \longrightarrow Z^{m} \times D^{r}
$$

given on a relative stable map $(C, f)$ by

$$
\left(x_{1}, \ldots, x_{m}, p_{1}, \ldots, p_{r}\right) \hookrightarrow C \longrightarrow{ }_{k} Z \longrightarrow Z .
$$

We will write $\mathrm{ev}_{i}: \mathcal{M Z} \longrightarrow Z$ or $\mathrm{ev}_{i}: \mathcal{M Z} \longrightarrow D$ to denote the evaluation map at one of the interior or boundary marked points.

Theorem 2.14 ([16]). $\mathcal{M Z}$ carries a virtual cycle of complex dimension

$$
\begin{aligned}
\operatorname{vdim} \mathcal{M Z}= & \sum_{v \in V(\Gamma)}(\operatorname{dim} Z-3)(1-g(v)) \\
& +<c_{1}(T Z)-D, d(v)>+r+m .
\end{aligned}
$$

2.2. Stack of maps to rubber. In constructing $\mathcal{M}(\mathcal{Z}, \Gamma)$, we had to consider stable maps to ${ }_{k} Z$ which was $Z$ union a chain of $P$ s. It is useful to consider stable maps to the chain of $P$ s subject to automorphisms. We call these maps to rubber.

Let $X$ be a projective manifold and $L$ a line-bundle on $X$. Let $P=$ $\mathbb{P}_{X}\left(L \oplus 1_{X}\right)$, and let $X_{0}$ and $X_{\infty}$ denote the zero and infinity sections. We study stable maps to $P$ relative to $X_{0}$ and $X_{\infty}$ where we mod out by a $\mathbb{C}^{*}$-factor that dilates the fibers. Again, the target $P$ may degenerate.

Definition 2.15. Let ${ }_{k} P$ be the union of $k+1$ copies of $P$,

$$
{ }_{k} P=P_{0} \sqcup_{X} P_{1} \sqcup_{X} \cdots \sqcup_{X} P_{k}
$$

gluing $X_{0} \subset P_{i}$ to $X_{\infty} \subset P_{i+1}$ for $i=0, \ldots, k-1$.

${ }_{k} P$ has distinguished divisors $D_{\infty}=X_{\infty} \subset P_{0}$ and $D_{0}=X_{0} \subset P_{k}$.

Definition 2.16. Let $\operatorname{Aut}\left({ }_{k} P\right)=\left(\mathbb{C}^{*}\right)^{k+1}$ act on ${ }_{k} P$ by dilating fibers of $P \longrightarrow X$.

Definition 2.17. A rubber topological type $\Gamma$ is the following data:

(1) a finite set $V(\Gamma)$;

(2) A genus assignment

$$
g: V(\Gamma) \longrightarrow \mathbb{Z}_{\geq 0}
$$

(3) A degree assignment

$$
d: V(\Gamma) \longrightarrow B_{1}(X)=A_{1}(X) / \sim_{\text {alg }} ;
$$

(4) Sets $R_{0}=\left\{1, \ldots, r_{0}\right\}, R_{\infty}=\left\{1, \ldots, r_{\infty}\right\}$ labeling boundary marked points together with a function assigning boundary marked points to elements of $V(\Gamma)$

$$
\begin{gathered}
a_{0}: R_{0} \longrightarrow V(\Gamma) \\
a_{\infty}: R_{\infty} \longrightarrow V(\Gamma) ;
\end{gathered}
$$


(5) A multiplicity assignment for boundary marked points

$$
\begin{aligned}
\mu^{0} & : R_{0} \longrightarrow \mathbb{Z}_{\geq 1} \\
\mu^{\infty} & : R_{\infty} \longrightarrow \mathbb{Z}_{\geq 1} ;
\end{aligned}
$$

(6) A set $M=\{1, \ldots, m\}$ labeling interior marked points together with an assignment

$$
a_{M}: M \longrightarrow V(\Gamma) .
$$

We may write $R_{A 0}, R_{A \infty}, M_{A}$ for $R_{0}, R_{\infty}$, and $M$, respectively.

Definitions of maps to ${ }_{k} P$ are analogous to maps to ${ }_{k} Z$ with $Z$ s replaced with $P$ s and the following modifications: the degree assignment is

$$
d(v) \in B_{1}(X)
$$

there are marked points

$$
\left(x_{1}, \ldots, x_{m}, p_{1}^{0}, \ldots, p_{r_{0}}^{0}, p_{1}^{\infty}, \ldots, p_{r_{\infty}}^{\infty}\right) \subset C
$$

so that

$$
\begin{aligned}
f^{*} D_{0} & =\sum_{i \in R_{0}} \mu^{0}(i) p_{i}^{0} \\
f^{*} D_{\infty} & =\sum_{i \in R_{\infty}} \mu^{\infty}(i) p_{i}^{\infty} .
\end{aligned}
$$

The notion of pre-deformable is directly analogous to the relative case. Stability is also analogous with $\operatorname{Aut}\left({ }_{k} P\right)$ substituted for $\operatorname{Aut}\left({ }_{k} Z\right)$.

Theorem 2.18. For a rubber topological type $\Gamma$, there is a proper DeligneMumford stack $\mathcal{M A}=\mathcal{M}(\mathcal{A}, \Gamma)$ parameterizing isomorphism classes of predeformable stable maps to ${ }_{k} P$ for varying $k$.

Theorem 2.19. $\mathcal{M A}$ carries a virtual cycle of complex dimension

$$
\begin{aligned}
\operatorname{vdim} \mathcal{M A}= & \sum_{v \in V(\Gamma)}\left((\operatorname{dim} X-2)(1-g(v))+<c_{1}(T X), d(v)>\right) \\
& +r_{0}+r_{\infty}+m-1 .
\end{aligned}
$$

The multiplicity condition relates the multiplicities at $D_{0}$ and $D_{\infty}$, to the degree:

Lemma 2.20. If $\mathcal{M}(\mathcal{A}, \Gamma)$ is non-empty then for each $v \in V(\Gamma)$ we have

$$
\sum_{p \in a_{0}^{-1}(v)} \mu^{0}(v)-\sum_{p \in a_{\infty}^{-1}(v)} \mu^{\infty}(v)=<c_{1}(L), d(v)>.
$$

Proof. One uses $\left[X_{0}\right]=\left[X_{\infty}\right]+\pi^{*} c_{1}(L)$ for each copy of $P$ in the target. 
We have analogous evaluation maps $\mathrm{ev}_{i}$ at the interior and boundary marked points (mapping to $D_{0}$ and $D_{\infty}$ ).

Definition 2.21. The evaluation map on $\mathcal{M A}=\mathcal{M}(\mathcal{A}, \Gamma)$ is

$$
\mathrm{Ev}: \mathcal{M A} \longrightarrow X^{m} \times X^{r_{0}} \times X^{r_{\infty}} .
$$

This moduli stack is constructed from a moduli functor by considering stable maps to targets modeled on a sequence of spaces and divisors $\left(A[n], D_{0}[n], D_{\infty}[n]\right)$ where

$$
\begin{aligned}
A[0] & =P \\
D_{0}[0] & =X_{0} \\
D_{\infty}[0] & =X_{\infty} \\
A[n] & =\mathrm{Bl}_{D_{0}[n-1] \times\{0\}}\left(A[n-1] \times \mathbb{A}^{1}\right),
\end{aligned}
$$

where $D_{0}[n]$ is the proper transform of $D_{0}[n-1] \times \mathbb{A}^{1}$, and $D_{\infty}[n]$ is the inverse image of $D_{\infty}[n-1] \times \mathbb{A}^{1} \cdot A[n] / X$ possesses a $\left(\mathbb{C}^{*}\right)^{n+1}$ group of automorphisms.

Definition 2.22. A split map in $\mathcal{M A}$ is a map $f: C \rightarrow_{k} P$ where $k \geq 1$, that is, a map whose target is not smooth.

Definition 2.23. For a map $f: C \rightarrow{ }_{k} P$ in $\mathcal{M A}$, the irreducible components of $C$ that are mapped to $P_{k}$ are said to be the top components while the components of $C$ that are mapped to $P_{0}$ are said to be the bottom components.

We should explain our top/bottom convention. In $Z$, moving towards $D$ is considered moving towards the top. In $P, D_{0}$ is considered the top while $D_{\infty}$ is the bottom. This slightly odd convention makes sense in that the most natural choice for $(X, L)$ is $\left(D, N_{D / Z}\right)$. In this case, the zero section of $P$ is identified with $D$ and the normal bundle to $D_{0}$ in $P$ is equal to the normal bundle to $D$ in $Z$. Therefore, $D_{0} \subset P$ like $D \subset Z$ is on top.

2.3. Trivial cylinders. We will single out certain connected components of curves parameterized by $\mathcal{M A}$. These are the trivial cylinders which will be significant when we encode the data of the moduli space into generating functions.

Definition 2.24. Let $\Gamma$ be a rubber topological type. An element $v \in V(\Gamma)$ is said to correspond to a trivial cylinder of degree $r$ if

(1) $g(v)=0$

(2) $d(v)=0$

(3) $a_{0}^{-1}(v)$ is a single point,

(4) $a_{\infty}^{-1}(v)$ is a single point, 
(5) $\mu^{0}\left(a_{0}^{-1}(v)\right)=\mu^{\infty}\left(a_{\infty}^{-1}(v)\right)=r$

(6) $a_{M}^{-1}(v)$ is empty.

A trivial cylinder corresponds to a connected component of the domain of a map $f \in \mathcal{M A}$. This map is from a chain of $k+1 \mathbb{P}^{1}$ s to ${ }_{k} P$, where each $\mathbb{P}^{1}$ is mapped to a fiber of $P_{i} \rightarrow X$ is of degree $r$ and is totally ramified at $X_{0}$ and $X_{\infty}$.

Note that if $V(\Gamma)$ has a single element corresponding to a trivial cylinder, then all rubber maps of type $\Gamma$ are invariant under the $\left(\mathbb{C}^{*}\right)^{k+1}$-action that dilates the fibers of ${ }_{k} P$. Therefore, there are no stable rubber maps and the moduli space is empty. This does not rule out maps of a type $\Gamma$ which have a component that is a trivial cylinder. In fact, one can add a trivial cylinder component to any family.

2.4. Gluing moduli stacks. Consider a projective manifold $Z$, together with a smooth divisor $D$. We will consider a relative moduli stack $\mathcal{M}\left(\mathcal{Z}, \Gamma_{Z}\right)$ corresponding to $(Z, D)$ and a rubber moduli stack $\mathcal{M}\left(\mathcal{A}, \Gamma_{A}\right)$ corresponding to $(X, L)$, where $X=D$ and $L=N_{D / Z}$, the normal bundle to $D$ in $Z$. One can join maps in $\mathcal{M}\left(\mathcal{Z}, \Gamma_{Z}\right)$ to maps in $\mathcal{M}\left(\mathcal{A}, \Gamma_{A}\right)$ if certain conditions are met. Likewise under particular conditions, one can join maps in $\mathcal{M}\left(\mathcal{A}, \Gamma_{A_{b}}\right)$ to maps in $\mathcal{M}\left(\mathcal{A}, \Gamma_{A_{t}}\right)$. We make these conditions precise below.

Definition 2.25. Let $\Gamma_{Z}$ be a relative topological type and $\Gamma_{A}$ be a rubber topological type. Suppose that $L: R_{Z} \rightarrow R_{A \infty}$ is a bijection from the labeling sets for boundary marked points in $\Gamma_{Z}$ to the labeling sets for boundary marked points mapping to $D_{\infty}$ in $\Gamma_{A}$ so that

$$
\mu_{Z}(q)=\mu_{A}^{\infty}(L(q)) \text {. }
$$

Let

$$
J: M_{Z} \sqcup M_{A} \longrightarrow\left\{1, \ldots,\left|M_{Z}\right|+\left|M_{A}\right|\right\}
$$

be a bijection between the labeling sets of the interior marked points and a set of $\left|M_{Z}\right|+\left|M_{A}\right|$ elements. We call the data $\left(\Gamma_{A}, \Gamma_{Z}, L, J\right)$ a type join quadruple.

Colloquially, we have matched boundary marked points on $\Gamma_{Z}$ and $\Gamma_{A}$ with the same multiplicity and numbered the marked points in $M_{Z} \sqcup M_{A}$.

Definition 2.26. Define the type join $\Gamma=\Gamma_{A} *_{L, J} \Gamma_{Z}$ to be the following relative topological type. Let the graph $\Delta$ be obtained by taking as vertices the set $V\left(\Gamma_{Z}\right) \sqcup V\left(\Gamma_{A}\right)$ and for every $q \in R_{Z}$ placing an edge between the vertices corresponding to $q$ and $L(q)$. The elements of $V(\Gamma)$ are the connected components of $\Delta$. Let $b_{Z}: V\left(\Gamma_{Z}\right) \rightarrow V(\Gamma)$ and $b_{A}: V\left(\Gamma_{A}\right) \rightarrow V(\Gamma)$ be the functions taking elements of $V\left(\Gamma_{A}\right)$ and $V\left(\Gamma_{A}\right)$ to the components in $\Delta$ containing them. For $v \in V(\Gamma)$, let $\Delta_{v}$ be the connected component of $\Delta$ 
corresponding to $v$. Define the data for $\Gamma_{A} *_{L, J} \Gamma_{Z}$ as follows:

(1) $g(v)=\left(\sum_{w \in b_{Z}^{-1}(v)} g(w)\right)+\left(\sum_{w \in b_{A}^{-1}(v)} g(w)\right)+h^{1}\left(\Delta_{v}\right)$;

(2) $d(v)=\left(\sum_{w \in b_{Z}^{-1}(v)} d(w)\right)+\left(\sum_{w \in b_{A}^{-1}(v)} I_{*} d(w)\right)$, where $I: X \rightarrow Z$ is the inclusion and $I_{*}: B_{1}(X) \rightarrow B_{1}(Z)$ is the induced map;

(3) $R=R_{A 0}$ with $a_{R}: R \rightarrow V(\Gamma)$ given by

$$
a_{R}=b_{A} \circ a_{0}
$$

(4) $\mu: R \longrightarrow \mathbb{Z}_{\geq 1}$ given by

$$
\mu=\mu^{0}
$$

(5) $M=\left\{1, \ldots,\left|M_{Z}\right|+\left|M_{A}\right|\right\}$ with assignment function $a: M \rightarrow V(\Gamma)$ given for $k \in J\left(M_{Z}\right)$ by

$$
a(k)=b_{Z} \circ a_{M_{Z}} \circ J^{-1}
$$

and for $k \in J\left(M_{A}\right)$ by

$$
a(k)=b_{A} \circ a_{M_{A}} \circ J^{-1} .
$$

Example 2.27. As an example, take $\Gamma_{Z}$ to be

(1) $V\left(\Gamma_{Z}\right)=\{a, b, c\}$,

(2) $g(a)=1, g(b)=2, g(c)=0$,

(3) $R_{Z}=\{1,2,3,4\}, a_{R}(1)=a, a_{R}(2)=a, a_{R}(3)=b, a_{R}(4)=c$,

(4) $\mu(1)=2, \mu(2)=3, \mu(3)=4, \mu(4)=17$,

(5) $M_{Z}=\{1,2\}, a_{M}(1)=a, a_{M}(2)=b$.

Take $\Gamma_{A}$ to be

(1) $V\left(\Gamma_{A}\right)=\{y, z\}$

(2) $g(y)=0, g(z)=7$,

(3) $R_{A \infty}=\{1,2,3,4\}, a_{\infty}(1)=y, a_{\infty}(2)=y, a_{\infty}(3)=y, a_{\infty}(4)=z$,

$R_{A 0}=\{1,2\}, a_{0}(1)=y, a_{0}(2)=z$,

(4) $\mu^{\infty}(1)=2, \mu^{\infty}(2)=3, \mu^{\infty}(3)=4, \mu^{\infty}(4)=17$, $\mu^{0}(1)=3, \mu^{0}(2)=28$,

(5) $M_{A}=\{1\}, a_{M}(1)=z$.

Let $L: R_{Z} \rightarrow R_{A \infty}$ be given by $L(1)=1, L(2)=2, L(3)=3, L(4)=4$ and $J: M_{Z} \sqcup M_{A} \rightarrow\{1,2,3\}$, where $\left.J\right|_{M_{Z}}(1)=1,\left.J\right|_{M_{Z}}(2)=2,\left.J\right|_{M_{A}}(1)=3$.

Then the type join $\Gamma_{A} *_{L, J} \Gamma_{Z}$ is given by

(1) $V\left(\Gamma_{A} *_{L, J} \Gamma_{Z}\right)=\{e, f\}$

(2) $g(e)=4, g(f)=7$,

(3) $R_{Z}=\{1,2\}, a_{R}(1)=e, a_{R}(2)=f$,

(4) $\mu(1)=3, \mu(2)=28$,

(5) $M_{Z}=\{1,2,3\}, a_{M}(1)=e, a_{M}(2)=e, a_{M}(3)=f$.

This is illustrated in Figure 1. 


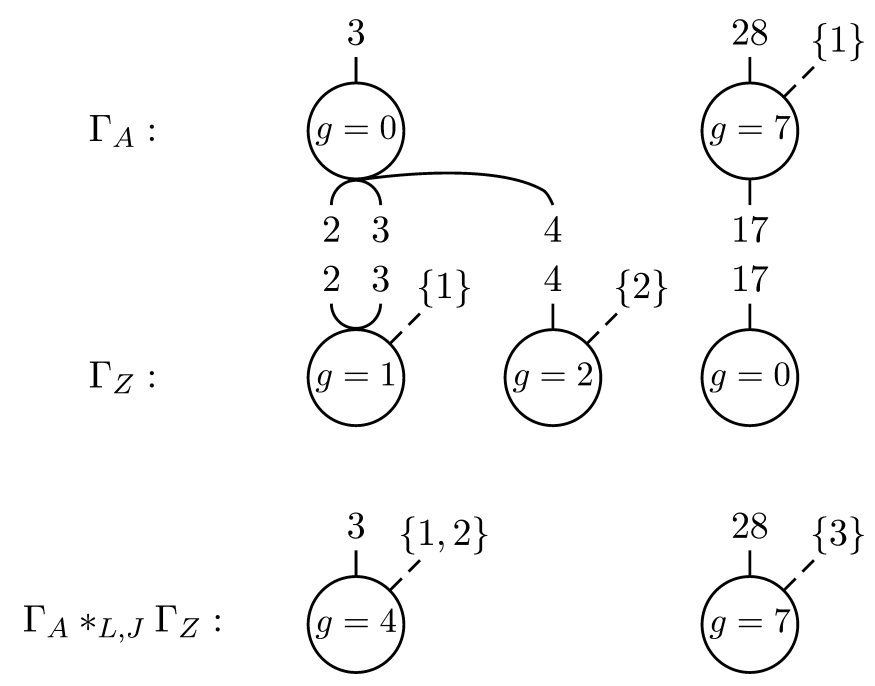

Figure 1. The type join $\Gamma_{A} *_{L, J} \Gamma_{Z}$ of the example.

Given $\left(\Gamma_{A}, \Gamma_{Z}, L, J\right)$, consider the evaluation map at the boundary marked points on $\mathcal{M}\left(\mathcal{Z}, \Gamma_{Z}\right)$ followed by a map $L_{*}: D^{r} \rightarrow D^{r}$ which reorders the products of $D^{r}$ according to $L$,

$$
L_{*} \circ \operatorname{Ev}_{R}: \mathcal{M}\left(\mathcal{Z}, \Gamma_{Z}\right) \longrightarrow D^{r} \longrightarrow D^{r}
$$

and the evaluation map at the boundary marked points mapping to $D_{\infty} \cong X$ on $\mathcal{M}\left(\mathcal{A}, \Gamma_{A}\right)$,

$$
\operatorname{Ev}_{R_{\infty}}: \mathcal{M}\left(\mathcal{A}, \Gamma_{A}\right) \longrightarrow D^{r}
$$

Theorem 2.28 ([15]). There is a morphism

$$
\Phi_{\Gamma_{A}, \Gamma_{Z}, L, J}: \mathcal{M}\left(\mathcal{A}, \Gamma_{A}\right) \times_{D^{r}} \mathcal{M}\left(\mathcal{Z}, \Gamma_{Z}\right) \longrightarrow \mathcal{M}\left(\mathcal{Z}, \Gamma_{A} *_{L, J} \Gamma_{Z}\right) .
$$

Definition 2.29. Let the stack $\mathcal{M}\left(\mathcal{A} \sqcup \mathcal{Z}, \Gamma_{A} \sqcup_{L, J} \Gamma_{Z}\right)$ be the image stack of $\Phi$ in $\mathcal{M}\left(\mathcal{Z}, \Gamma_{A} *_{L, J} \Gamma_{Z}\right)$.

Definition 2.30. An automorphism of $R_{Z}$ is a permutation

$$
\sigma: R_{Z} \longrightarrow R_{Z}
$$

so that $\mu_{Z}(\sigma(i))=\mu_{Z}(i)$ and $a_{R}(\sigma(i))=a_{R}(i)$. The group of all such automorphisms is denoted by $\operatorname{Aut}_{\Gamma_{Z}}\left(R_{Z}\right)$. Likewise, we $\operatorname{define}^{\operatorname{Aut}_{\Gamma_{A}}}\left(R_{A 0}\right)$ and $\operatorname{Aut}_{\Gamma_{A}}\left(R_{A \infty}\right)$.

Given $L: R_{Z} \rightarrow R_{A \infty}$, we may define $\operatorname{Aut}_{\Gamma_{A}, \Gamma_{Z}, L}\left(R_{A \infty}, R_{Z}\right)$ as the subgroup of $\operatorname{Aut}_{\Gamma_{Z}}\left(R_{Z}\right) \times \operatorname{Aut}_{\Gamma_{A}}\left(R_{A \infty}\right)$ such that for $(\sigma, \tau) \in \operatorname{Aut}_{\Gamma_{Z}}\left(R_{Z}\right) \times$ $\operatorname{Aut}_{\Gamma_{A}}\left(R_{A \infty}\right)$, we have $L(\sigma(i))=\tau(L(i))$ for $1 \leq i \leq\left|R_{Z}\right|$. 
Lemma 2.31 ([15], Proposition 4.13). $\Phi$ is finite and étale onto its image of degree equal to

$$
\left|\operatorname{Aut}_{\Gamma_{A}, \Gamma_{Z}, L}\left(R_{A \infty}, R_{Z}\right)\right|
$$

at every integral substack of $\mathcal{M}\left(\mathcal{A} \sqcup \mathcal{Z}, \Gamma_{A} \sqcup_{L, J} \Gamma_{Z}\right)$.

Definition 2.32. Let $\Upsilon=\left(\Gamma_{A}, \Gamma_{Z}, L, J\right)$ be a type join quadruple. The boundary multiplicity $m(\Upsilon)$ is

$$
m(\Upsilon)=\prod_{i \in R_{Z}} \mu_{Z}(i)
$$

Definition 2.33. Two quadruples are said to be join-equivalent if they give the same image under $\Phi$.

Proposition 2.34. Consider a join-equivalence class of quadruples

$$
[\Upsilon]=\left[\left(\Gamma_{A}, \Gamma_{Z}, L, J\right)\right] .
$$

Let $N=\mathcal{M}\left(\mathcal{A} \sqcup \mathcal{Z}, \Gamma_{A} \sqcup_{L, J} \Gamma_{Z}\right)$, and

$$
M_{\Upsilon}=\coprod_{\left(\Gamma_{A}^{\prime}, \Gamma_{Z}^{\prime}, L^{\prime}, J^{\prime}\right)} \mathcal{M}\left(\mathcal{A}, \Gamma_{A}^{\prime}\right) \times_{D^{r}} \mathcal{M}\left(\mathcal{Z}, \Gamma_{Z}^{\prime}\right)
$$

where the disjoint union is over quadruples join-equivalent to $\Upsilon$. Then $\Phi_{[\Upsilon]}: M \rightarrow N$ is an étale map of degree

$$
\left|M_{Z}\right| !\left|M_{A}\right| !\left(\left|R_{Z}\right| !\right)^{2}
$$

Proof. This follows from the previous lemma and the fact that there are

$$
\left|M_{Z}\right| !\left|M_{A}\right| ! \frac{\left(\left|R_{Z}\right| !\right)^{2}}{\left|\operatorname{Aut}_{\Gamma_{A}, \Gamma_{Z}, L}\left(R_{A \infty}, R_{Z}\right)\right|}
$$

elements in $\left(\Gamma_{Z}, \Gamma_{A}, L, J\right)$ s type join-equivalence class.

Likewise, we may define type join for rubber topological types, $\Gamma_{t}, \Gamma_{b}$ (where $t$ and $b$ stand for top and bottom). Let $L: R_{A_{b} 0} \rightarrow R_{A_{t} \infty}$ be a bijective function satisfying

$$
\mu_{b}^{0}(q)=\mu_{t}^{\infty}(L(q))
$$

Let

$$
J: M_{A_{b}} \sqcup M_{A_{t}} \longrightarrow\left\{1, \ldots,\left|M_{A_{b}}\right|+\left|M_{A_{t}}\right|\right\}
$$

be a bijective map. Then we define the type join, a rubber topological type $\Gamma=\Gamma_{t} *_{L, J} \Gamma_{b}$ as above, except that instead of condition (3) above, we have

$$
\begin{aligned}
R_{0} & =R_{A_{t} 0}, \quad a_{0}=b_{A_{t}} \circ a_{t 0} \\
\mu^{0} & =\mu_{t}^{0} \\
R_{\infty} & =R_{A_{b} \infty}, \quad a_{\infty}=b_{A_{b}} \circ a_{b \infty} \\
\mu^{\infty} & =\mu_{b}^{\infty} .
\end{aligned}
$$


Definition 2.35. Let $\Upsilon=\left(\Gamma_{A_{t}}, \Gamma_{A_{b}}, L, J\right)$ be a type join quadruple. Define $m(\Upsilon)$ by

$$
m(\Upsilon)=\prod_{i \in R_{A_{t}}} \mu_{t}^{\infty}(i)
$$

Now, let $r=\left|R_{A_{b} 0}\right|=\left|R_{A_{t} \infty}\right|$. Analogously to the above, we have

Theorem 2.36 ([15]). There is a morphism

$$
\Phi: \mathcal{M}\left(\mathcal{A}, \Gamma_{A_{t}}\right) \times{ }_{D^{r}} \mathcal{M}\left(\mathcal{A}, \Gamma_{A_{b}}\right) \longrightarrow \mathcal{M}\left(\mathcal{A}, \Gamma_{A_{t}} *_{L, J} \Gamma_{A_{b}}\right)
$$

étale of degree $\left|\operatorname{Aut}_{\Gamma_{A_{t}}, \Gamma_{A_{b}}, L}\left(R_{A_{t} \infty}, R_{A_{b} 0}\right)\right|$.

Corollary 2.37. Consider a moduli stack $N=\mathcal{M}\left(\mathcal{A}, \Gamma_{A_{t}} \sqcup_{L, J} \Gamma_{A_{b}}\right)$. Let

$$
M=\coprod_{\left(\Gamma_{A_{t}}^{\prime}, \Gamma_{A_{b}}^{\prime}, L^{\prime}, J^{\prime}\right)} \mathcal{M}\left(\mathcal{A}, \Gamma_{A_{t}}^{\prime}\right) \times_{D^{r}} \mathcal{M}\left(\mathcal{A}, \Gamma_{A_{b}}^{\prime}\right)
$$

where the disjoint union over $\left(\Gamma_{A_{t}}, \Gamma_{A_{b}}, L, J\right)$ s join-equivalence class. Then $M \rightarrow N$ is an étale map of degree

$$
\left|M_{A_{b}}\right| !\left|M_{A_{t}}\right| !\left(\left|R_{A_{b} 0}\right| !\right)^{2} .
$$

2.5. Line-bundles on moduli stacks. The moduli stacks carry linebundles with particular geometric meaning.

Given a relative topological type $\Gamma_{Z}, \mathcal{M}\left(\mathcal{Z}, \Gamma_{Z}\right)$ has canonically defined line-bundles:

(1) A line bundle Dil that has a section whose zero stack is supported on all split maps;

(2) For $i$ is a distinguished interior marked point, a line bundle $\mathrm{L}_{i \text {,ext }}$ that has a section whose zero stack is supported on split maps where $i$ lies on an extended component.

It will be outlined below that $c_{1}$ (Dil) $\cap[\mathcal{M Z}]^{\text {vir }}$ is (with multiplicity) the locus of split maps and $c_{1}\left(\mathrm{~L}_{i, \text { ext }}\right) \cap[\mathcal{M Z}]^{\text {vir }}$ is the locus of split maps with $i$ on an extended component.

For $\Gamma_{A}, \mathcal{M}\left(\mathcal{A}, \Gamma_{A}\right)$ has the following line-bundles:

(1) The target cotangent line-bundle at $X_{0}, \mathrm{~L}^{0}$;

(2) The target cotangent line-bundle at $X_{\infty}, \mathrm{L}^{\infty}$;

(3) The split bundle, Split which has a section whose zero stack is supported on all split maps (Definition 2.22);

(4) For $i$, a distinguished interior marked point, the not-top bundle with

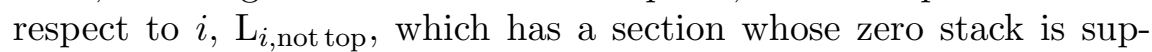
ported on split maps where the $i$ th marked point is not on a top component; 
(5) Analogously to (4), the not-bottom bundle with respect to $i, \mathrm{~L}_{i \text {,not bot }}$, which has a section whose zero stack is supported on split maps where the $i$ th marked point is not on a bottom component.

$\mathrm{L}^{0}$, which is defined in terms of an atlas, has an intuitive description. Given a map to rubber, $f: C \rightarrow_{k} P$ in $\mathcal{M A}$, let $\widehat{C}$ be the union of components of $C$ mapping to $P_{k}$. There is a $\mathbb{C}^{*}$ family of maps $\widehat{f}: \widehat{C} \rightarrow P$ that occur as the restriction of $\mathrm{f}$. These $\mathbb{C}^{*}$ families fit together to give a $\mathbb{C}^{*}$ bundle, and an associated $\mathbb{C}$ bundle is $\mathrm{L}^{0} . \mathrm{L}^{\infty}$ is the analogous bundle where we consider the bottom component. The first Chern class of $\mathrm{L}^{0}, c_{1}\left(\mathrm{~L}^{0}\right)=\Psi_{0}$, the target $\Psi$ class of $[\mathbf{3}]$.

$\mathrm{L}^{0}$ and $\mathrm{L}^{\infty}$ can be given an interpretation in the stack of rational sausages, the substack of $\mathfrak{M}_{0,2}$ consisting of pre-stable curves so that the two marked points lie on different sides of every node. $L^{0}$ and $L^{\infty}$ are equal to the pullbacks of the cotangent line classes at the two marked points. See [9] for an elaboration.

Theorem $2.38([\mathbf{1 1}])$. The line-bundles satisfy the following relations. On $\mathcal{M}\left(\mathcal{Z}, \Gamma_{Z}\right)$,

and on $\mathcal{M}\left(\mathcal{A}, \Gamma_{A}\right)$,

$$
e v_{i}^{*} \mathcal{O}(D)=\mathrm{L}_{i, \text { ext }},
$$

$$
\begin{aligned}
\mathrm{L}^{0} \otimes \mathrm{L}^{\infty} & =\text { Split } \\
\mathrm{L}^{0} \otimes \mathrm{ev}_{i}^{*} L^{\vee} & =\mathrm{L}_{i, \text { not top }} \\
\mathrm{L}^{\infty} \otimes \mathrm{ev}_{i}^{*} L & =\mathrm{L}_{i, \text { not bot }} .
\end{aligned}
$$

If we consider the stack of rational sausages where $\mathrm{L}^{0}$ and $\mathrm{L}^{\infty}$ are the restriction of $\psi$ classes on $\mathfrak{M}_{0,2}$, then (2.1) is the pullback of the genus 0 recursion relation of Lee and Pandharipande [13]. A proof of the enumerative consequences of (2.2) and (2.3) is given in Section 7. This proof is sufficient to establish the results of Section 6 .

\section{Generating functions}

An important idea in Gromov-Witten theory, originating [24], is that of organizing invariants in generating functions. Relations satisfied by the invariants become differential equations for the generating function. In this section, we define generating functions for relative and rubber invariants motivated by SFT [2].

3.1. Relative potential. Let us consider a pair $(Z, D)$, where $Z$ is a projective manifold and $D \subset Z$ is a smooth divisor on $Z$. The generating function of the relative invariants takes values in a particular graded algebra.

Let us specify the following data: an Euler characteristic, $\chi$; a curve class (up to algebraic equivalence), $d \in B_{1}(Z)$; a number of interior marked points, 
$m$; a number of boundary marked points, $r$; and a $r$-tuple of multiplicities to $D,\left(s_{1}, s_{2}, \ldots, s_{r}\right)$. Consider the set $\Xi$ of relative topological types $\Gamma$ so that

(1) $\sum_{v} d(v)=d$

(2) $\sum_{v}(2-2 g(v))=\chi$,

(3) $|M|=m$,

(4) $|R|=r$,

(5) $(\mu(1), \mu(2), \ldots, \mu(r))=\left(s_{1}, s_{2}, \ldots, s_{r}\right)$.

To each $\Gamma$, we associate the moduli space $\mathcal{M Z}=\mathcal{M}(\mathcal{Z}, \Gamma)$ which has evaluation map,

$$
\mathrm{Ev}: \mathcal{M Z} \longrightarrow(Z)^{m} \times(D)^{r}
$$

Definition 3.1. Given cohomology classes

$$
e_{1}, \ldots, e_{n} \in H^{*}(Z)
$$

and

$$
c_{1}, \ldots, c_{r} \in H^{*}(D)
$$

define the correlator by

$$
\begin{aligned}
\left\langle e_{1}\right. & \left.\ldots e_{n}, c_{1} \ldots c_{r}\right\rangle_{\chi, d,\left(s_{1}, \ldots, s_{r}\right)} \\
\quad & =\sum_{\Gamma \in \Xi} \operatorname{Ev}^{*}\left(e_{1} \times \cdots \times e_{n} \times c_{1} \times \cdots \times c_{r}\right) \cap[\mathcal{M}(\mathcal{Z}, \Gamma)]^{\mathrm{vir}}
\end{aligned}
$$

By stability considerations, the above sum over relative topological types is finite.

Let $e_{1}, e_{2}, \ldots, e_{l}$ be a homogeneous basis of $H^{*}(Z)$. Let $c_{1}, c_{2}, \ldots, c_{k}$ be a homogeneous basis of $H^{*}(D)$. Let $\mathbb{Q}\left[B_{1}(Z)\right]$ be the group algebra on $B_{1}(Z)$.

Consider the graded super-commutative algebra over $\mathbb{Q}\left[B_{1}(Z)\right]$ freely generated by $\tilde{\hbar}^{-1}, \tilde{\hbar}, \theta_{1}, \theta_{2}, \ldots, \theta_{l}$, and, for every positive integer $n$, elements $\tilde{p}_{n, 1}, \tilde{p}_{n, 2}, \ldots, \tilde{p}_{n, k}$ with the following degrees:

$$
\begin{aligned}
\operatorname{deg} \tilde{\hbar} & =-2(\operatorname{dim} Z-3) \\
\operatorname{deg} \tilde{z}^{d} & =2\left\langle c_{1}(T X), d\right\rangle \\
\operatorname{deg} \theta_{i} & =2-\operatorname{deg} e_{i} \\
\operatorname{deg} \tilde{p}_{n, j} & =2-\operatorname{deg} c_{i}-2 j .
\end{aligned}
$$

$\hbar$ is a formal variable corresponding to one half of the Euler characteristic, $\tilde{z}^{d}$ to degree, $\theta_{i}$ to interior marked points that are mapped by the evaluation map to a cycle Poincare-dual to $e_{i}$, and $\tilde{p}_{n, j}$ to boundary marked points with multiplicity $n$ and mapped to a cycle Poincare-dual to $c_{j}$ on $D$.

We define $\mathcal{F}$ to be a partial completion of the above algebra where we allow Laurent series in $\tilde{\hbar}$ whose coefficients are polynomials in the $\tilde{p}$-variables whose coefficients are power series in the $\theta$ variables. 
Let $\mathcal{G}$ be the non-commutative algebra of power series in $\theta_{i} e_{i}$. Let $\mathcal{P}$ be the non-commutative algebra of power series in $\tilde{p}_{n, j} c_{j}$.

Define the $\mathcal{F}$-correlator to be

$$
\begin{aligned}
& \left\langle\theta_{i_{1}} e_{i_{1}} \ldots \theta_{i_{n}} e_{i_{n}}, \tilde{p}_{s_{1}, j_{1}} c_{j_{1}} \ldots \tilde{p}_{s_{r}, j_{r}} c_{j_{r}}\right\rangle_{\chi, d} \\
& \quad=\theta_{i_{1}} \ldots \theta_{i_{n}} \tilde{p}_{s_{1}, j_{1}} \ldots \tilde{p}_{s_{r}, j_{r}}\left\langle e_{i_{1}} \ldots e_{i_{n}}, c_{j_{1}} \ldots c_{j_{r}}\right\rangle_{\chi, d,\left(s_{1}, s_{2}, \ldots, s_{r}\right)} .
\end{aligned}
$$

Extend the $\mathcal{F}$-correlator multi-linearly to a map

$$
(,)_{g, d}: \mathcal{G} \otimes \mathcal{P} \longrightarrow \mathcal{F}
$$

Let $\tilde{\Gamma} \in \mathcal{G}, \tilde{P} \in \mathcal{P}$ be given by

$$
\begin{aligned}
& \tilde{\Gamma}=\sum_{l \geq 0} \frac{1}{l !}\left(\sum \theta_{i} e_{i}\right)^{l} \\
& \tilde{P}=\sum_{n} \frac{1}{n !}\left(\sum_{k, i} \tilde{p}_{k, i} c_{i}\right)^{n} .
\end{aligned}
$$

Definition 3.2. The relative potential of $(Z, D)$ is $F \in \mathcal{F}$ defined by

$$
F=\sum_{g \geq 0} \sum_{d \in B_{1}(Z)}\langle\tilde{\Gamma}, \tilde{P}\rangle_{g, d} \hbar^{g-1} z^{d}
$$

Note that $F$ is indeed in $\mathcal{F}$. The coefficient of $z^{A}$ is a polynomial in the $\tilde{p}$ variables because the number of $\tilde{p}$ variables is bounded by $A \cdot D$. By our choice of degrees for the formal variable and by the virtual dimension of $\mathcal{M Z}, F$ is a homogeneous element of degree of 0 . Our usage of $F$ disagrees with that of $[\mathbf{2}]$ because we consider disconnected stable maps.

Example 3.3. For the target $\left(\mathbb{P}^{1}, \infty\right)$, the relative potential is

$$
F=\exp \left(\hbar^{-1}\left(\frac{1}{2 !} \theta_{0}^{2} \theta_{1}+e^{\theta_{1}} p_{1,1} z\right)+\hbar^{0}\left(-\frac{1}{24} \theta_{1}\right)\right)
$$

where $\theta_{0}$ and $\theta_{1}$ are dual to the classes $\left[\mathbb{P}^{1}\right]$, [pt]. The $\hbar^{-1} \frac{1}{2 !} \theta_{0}^{2}$ corresponds to contracted rational curves, $\hbar^{-1} e^{\theta_{1}} p_{1,1} z$ to degree 1 rational curves with arbitrarily many marked points, and $-\hbar^{0} \frac{1}{24} \theta_{1}$ to contracted elliptic curves. The contracted contributions were computed by hand.

Example 3.4. For the target $\left(\mathbb{P}^{2}, L\right)$, the relative potential is

$$
F=\exp \left(F_{d=0}+F_{d \geq 1}\right),
$$


where $F_{d=0}$ and $F_{d \geq 1}$ correspond to connected degree 0 and to positive degree maps, respectively:

$$
\begin{aligned}
F_{d=0}= & -\hbar^{0} \frac{1}{8} \theta_{1}+\hbar^{0}\left(\frac{1}{2 !} \theta_{2} \theta_{0}^{2}+\frac{1}{2 !} \theta_{1}^{2} \theta_{0}\right) \\
F_{d \geq 1}= & \hbar^{-1} \theta_{2} p_{1,1} z+\hbar^{-1} \frac{\theta_{2}^{2}}{2 !} p_{1,0} z+\hbar^{-1} \frac{\theta_{2}^{3}}{3 !}\left(p_{2,1}+\hbar^{-1} \frac{1}{2 !} p_{1,1}^{2}\right) z^{2} \\
& +\frac{\theta_{2}^{4}}{4 !}\left(2 p_{2,0}+p_{1,1} p_{1,0}\right) z^{2}+\cdots
\end{aligned}
$$

where, for example, $\hbar^{-1} \theta_{2} p_{1,1} z$ corresponds to a degree 1 rational map with one interior marked point mapping to a specified point in $\mathbb{P}^{2}$ and one boundary marked point mapping to a specified point in $L$, and $\hbar^{-1} \frac{\theta_{2}^{4}}{4 !} p_{1,1} p_{1,0} z^{2}$ corresponds to a degree 2 rational map through four specified points in $\mathbb{P}^{2}$ with two boundary points of contact of order 1 to $L$, one at a specified point, the other free. The positive-degree terms were computed using the methods of Section 5 while the degree 0 terms were computed by hand.

3.2. The rubber potential. We can combine all intersection numbers arising from all rubber moduli spaces into a generating function. Let $L$ be a line-bundle over a projective manifold $X$. Choose an Euler characteristic $\chi$, $m$ interior marked points, $r_{0}+r_{\infty}$ boundary marked points. Fix a curve class $d \in B_{1}(X)$, a $r_{0}$-tuple of multiplicities $\left(s_{1}^{0}, \ldots, s_{r_{0}}^{0}\right)$ to $D_{0}$ at the $r_{0}$ boundary marked points, and a $r_{\infty}$-tuple of multiplicities $\left(s_{1}^{\infty}, \ldots, s_{r_{\infty}}^{\infty}\right)$ to $D_{\infty}$ at the $r_{\infty}$ boundary marked points. Let $\Xi$ be the set of rubber topological types $\Gamma$ with no elements of $V(\Gamma)$ associated to trivial cylinders so that

(1) $\sum_{v} d(v)=d$,

(2) $\sum_{v}(2-2 g(v))=\chi$,

(3) $|M|=m$,

(4) $\left|R_{0}\right|=r_{0}$,

(5) $\left(\mu^{0}(1), \mu^{0}(2), \ldots, \mu^{0}\left(r_{0}\right)\right)=\left(s_{1}^{0}, s_{2}^{0}, \ldots, s_{r_{0}}^{0}\right)$,

(6) $\left|R_{\infty}\right|=r_{\infty}$,

(7) $\left(\mu^{\infty}(1), \mu^{\infty}(2), \ldots, \mu^{\infty}\left(r_{\infty}\right)\right)=\left(s_{1}^{\infty}, s_{2}^{\infty}, \ldots, s_{r_{\infty}}^{\infty}\right)$.

We have evaluation maps at the marked points

$$
\mathrm{Ev}: \mathcal{M}(\mathcal{A}, \Gamma) \longrightarrow X^{m} \times X^{r_{0}} \times X^{r_{\infty}} .
$$

We also have the two line-bundles on $\mathcal{M A}, \mathrm{L}^{0}$ and $\mathrm{L}^{\infty}$. Given a cohomology class $c \in H^{*}\left(X^{m+r_{0}+r_{\infty}}\right)$, we consider intersection numbers of the form

$$
\left(c_{1}\left(\mathrm{~L}^{\infty}\right)^{l} \cup \operatorname{Ev}^{*}(c)\right) \cap[\mathcal{M} \mathcal{A}]^{\mathrm{vir}} .
$$

Definition 3.5. Given a curve class $d \in B_{1}(X)$, an Euler characteristic $\chi$, non-negative integers $l, m, r_{0}, r_{\infty}$, multiplicities $S^{0}=\left(s_{1}^{0}, \ldots, s_{r_{0}}^{0}\right), S^{\infty}=$ 


$$
\begin{aligned}
& \left(s_{1}^{\infty}, \ldots, s_{r_{\infty}}^{\infty}\right), \\
& c_{1}, \ldots, c_{m}, e_{1}^{0}, \ldots, e_{r_{0}}^{0}, e_{1}^{\infty}, \ldots, e_{r_{\infty}}^{\infty} \in H^{*}(X),
\end{aligned}
$$

a non-negative integer $m$, define the correlator

$$
\begin{aligned}
\left(c_{1}\right. & \left.\ldots c_{m}, e_{1}^{0} \ldots e_{r_{0}}^{0}, e_{1}^{\infty} \ldots e_{r_{\infty}}^{\infty}\right)_{\chi, d, S^{0}, S^{\infty}, l} \\
= & \sum_{\Gamma \in \Xi} \operatorname{deg}\left(c _ { 1 } ( \mathrm { L } ^ { \infty } ) ^ { l } \cup \operatorname { E v } ^ { * } \left(c_{1} \times \cdots \times c_{m} \times e_{1}^{0} \times \cdots \times e_{r_{0}}^{0}\right.\right. \\
& \left.\left.\quad \times e_{1}^{\infty} \times \cdots \times e_{r_{\infty}}^{\infty}\right) \cap[\mathcal{M}(\mathcal{A}, \Gamma)]^{\mathrm{vir}}\right) .
\end{aligned}
$$

We organize the correlators into a generating function which we call the rubber potential which takes values in a particular graded algebra, $\mathcal{R}$. Choose a homogeneous basis $c_{1}, \ldots, c_{k}$ for $H^{*}(X)$. We define elements $z^{A}$ for all $A \in$ $B_{1}(X), \hbar, \lambda, \beta_{1}, \ldots, \beta_{k}, p_{n, 1}, \ldots, p_{n, k}, q_{n, 1}, \ldots, q_{n, k}$ for all positive integers $n$. The elements are graded of the following degrees:

$$
\begin{aligned}
\operatorname{deg} z^{d} & =2\left\langle c_{1}(T X), d\right\rangle+2\left\langle c_{1}(L), d\right\rangle \\
\operatorname{deg} \hbar & =-2(\operatorname{dim} X-2) \\
\operatorname{deg} \lambda & =-2 \\
\operatorname{deg} \beta_{i} & =2-\operatorname{deg} c_{i} \\
\operatorname{deg} p_{n, i} & =2-\operatorname{deg} c_{i}-2 n \\
\operatorname{deg} q_{n, i} & =2-\operatorname{deg} c_{i}+2 n .
\end{aligned}
$$

Multiplication in the algebra is defined as follows. The $\hbar_{-}, \lambda-, \beta_{i}$-variables are taken to be super-central while the $p$ - and $q$-variables obey supercommutation relations

$$
\left[p_{n_{1}, i_{1}}, p_{n_{2}, i_{2}}\right]=0,\left[q_{n_{1}, i_{1}}, q_{n_{2}, i_{2}}\right]=0,\left[q_{n_{1}, i_{1}}, p_{n_{2}, i_{2}}\right]=n_{1} \delta_{n_{1}, n_{2}} g^{i_{1} i_{2}} \hbar,
$$

where $g^{i_{1} i_{2}}$ is the Poincare pairing on $H^{*}(X)$. Note that this algebra can be realized by writing $q_{n, i}$ as a differential operator

$$
q_{n, i}=n \hbar \sum_{j} g^{i j} \frac{\partial}{\partial p_{n, j}} .
$$

The multiplication keeps track of different ways of joining curves. Let us consider an example in a toy model of our algebra. Consider variables $p_{1}, p_{2}, p_{3}$ which are all of an even parity together with $q_{1}, q_{2}, q_{3}$ so

$$
q_{i}=\hbar \frac{\partial}{\partial p_{i}} .
$$

Then,

$\left(\hbar^{-1} p_{1} q_{1} q_{2}\right) *\left(\hbar^{-1} p_{1} p_{2} p_{3}\right)=p_{1} p_{3}+\hbar^{-2} p_{1}^{2} p_{2} p_{3} q_{1} q_{2}+\hbar^{-1} p_{1}^{2} p_{3} q_{1}+\hbar^{-1} p_{1} p_{2} p_{3} q_{2}$. 


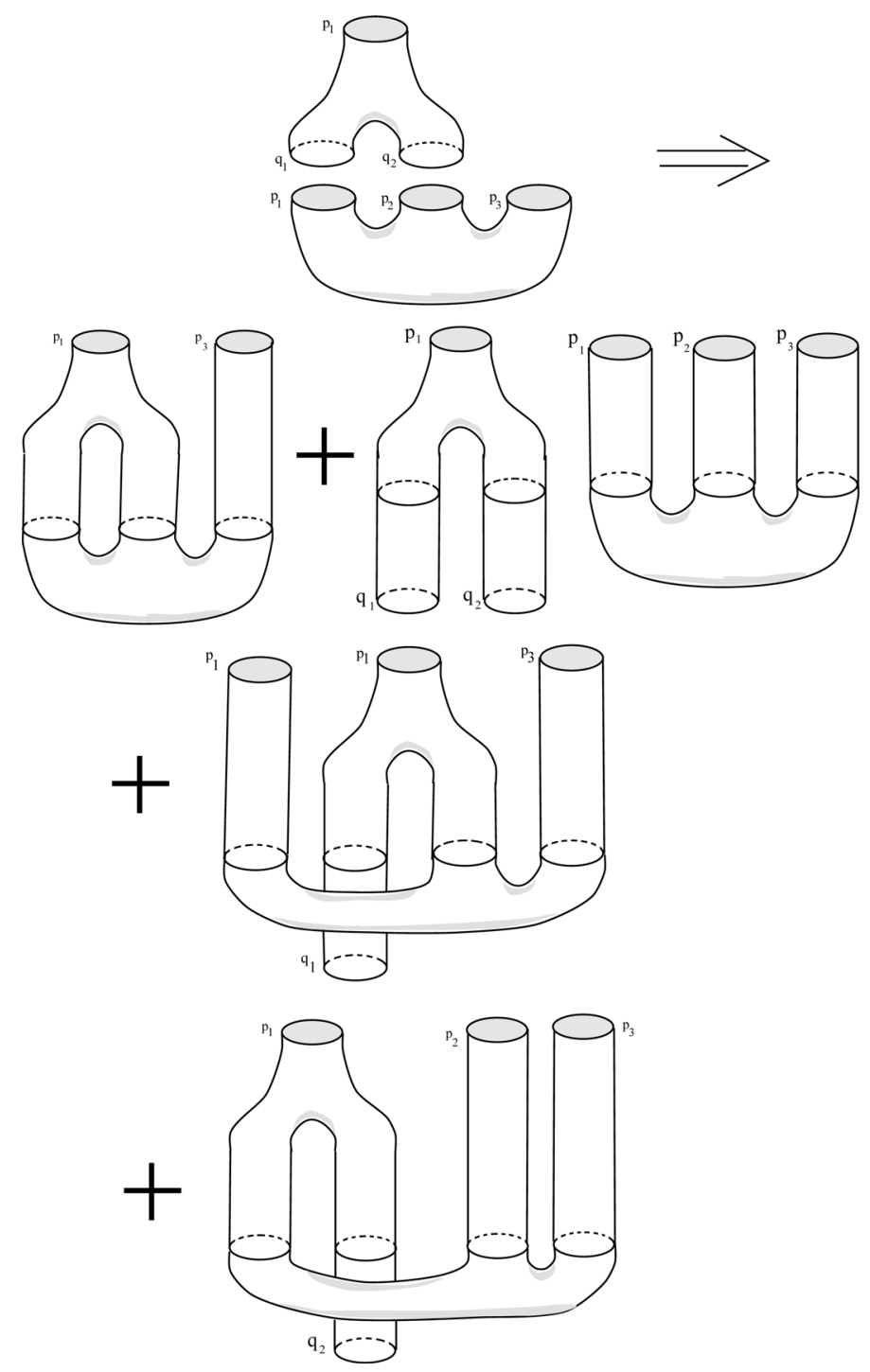

Figure 2. Geometric illustration of the multiplication in $\mathcal{R}$. This figure is borrowed from [2].

If we see $\hbar$ as a genus marker where a term with an Euler coefficient $\chi$ is marked with $\hbar^{-\frac{1}{2} \chi}$, this multiplication corresponds to the geometric situation illustrated in Figure 2.

The algebra $\mathcal{R}$ consists of Laurent series in $\hbar$ whose coefficients are power series in the $p$-variables whose coefficients are power series in the $\beta$-variables whose coefficients are polynomials in the $q$ - and $\lambda$-variables. 
Definition 3.6. The $\mathcal{R}$-correlator is

$$
\begin{aligned}
\left(\beta_{i_{1}} c_{i_{1}} \ldots \beta_{i_{m}} c_{i_{m}}, p_{s_{1}^{0}, j_{1}} c_{j_{1}} \ldots p_{s_{r_{0}}^{0}, j_{r_{0}}} c_{j_{r_{0}}}, q_{s_{1}^{\infty}, k_{1}} c_{k_{1}} \ldots q_{s_{r_{\infty}}^{\infty}, k_{r_{\infty}}} c_{k_{r_{\infty}}}\right)_{\chi, d, l} \\
=\beta_{i_{1}} \ldots \beta_{i_{m}} p_{s_{1}^{0}, j_{1}} \ldots p_{s_{r_{0}}^{0}, j_{r_{0}}} q_{s_{1}^{\infty}, k_{1}} \ldots q_{s_{r_{\infty}}^{\infty}, k_{r_{\infty}}} \\
\quad \cdot\left(c_{i_{1}} \ldots c_{i_{n}}, c_{j_{1}} \ldots c_{j_{r_{0}}}, c_{k_{1}} \ldots k_{r_{\infty}}\right)_{\chi, d,\left(s_{1}^{0}, \ldots, s_{r_{0}}^{0}\right),\left(s_{1}^{\infty}, \ldots, s_{r_{\infty}}^{\infty}\right), l}
\end{aligned}
$$

considered as an element of $\mathcal{R}$.

Let $\mathcal{B}, \mathcal{Q}, \mathcal{P}$ be the (non-commutative) power-series algebras freely generated by $\beta_{i} c_{i}, q_{n, i} c_{i}, p_{n, i} c_{i}$, respectively.

We can extend the $\mathcal{R}$-correlator by linearity to give a function

$$
(,,)_{\chi, d, m}: \mathcal{B} \otimes \mathcal{P} \otimes \mathcal{Q} \longrightarrow \mathcal{R} .
$$

Let $B \in \mathcal{B}, P \in \mathcal{P}, Q \in \mathcal{Q}$ be given by

$$
\begin{aligned}
& B=\sum_{l \geq 0} \frac{1}{l !}\left(\sum_{i} \beta_{i} c_{i}\right)^{l} \\
& P=\sum_{n} \frac{1}{n !}\left(\sum_{k, i} p_{k, i} c_{i}\right)^{n} \\
& Q=\sum_{n} \frac{1}{n !}\left(\sum_{k, i} q_{k, i} c_{i}\right)^{n} .
\end{aligned}
$$

Definition 3.7. The rubber potential $A$ is

$$
A=\sum_{\chi} \sum_{d \in B_{1}(X)} \sum_{l} \hbar^{-1 / 2 \chi} \frac{\lambda^{l}}{l !} z^{d}(B, P, Q)_{\chi, d, l} .
$$

Note that for a moduli stack $\mathcal{M A}$ to be non-empty, by Lemma 2.20, the multiplicities must satisfy

$$
s_{1}^{0}+\cdots+s_{r_{0}}^{0}-s_{1}^{\infty}-\cdots-s_{r_{\infty}}^{\infty}=\left\langle c_{1}(L), d\right\rangle
$$

so

$$
r_{\infty} \leq s_{1}^{0}+\cdots+s_{0}^{r_{0}}-\left\langle c_{1}(L), d\right\rangle .
$$

Therefore, the rubber potential satisfies the polynomiality in $q$ condition to lie in $\mathcal{R}$. The rubber potential $A$ is homogeneous of degree 2 .

Definition 3.8. The rubber potential without powers of $\mathrm{L}^{\infty}$ is given by $A_{\lambda=0}$. 
Example 3.9. The rubber potential of $(X, L)=\left(\mathrm{pt}, 1_{\mathrm{pt}}\right)$ without powers of $L^{\infty}$ is

$$
A_{\lambda=0}=\hbar^{-1}\left(\frac{1}{3 !} \theta_{0}^{3}+\frac{1}{2} \sum_{k, l \geq 1}\left(p_{k+l} q_{k} q_{l}+p_{k} p_{l} q_{k+l}\right)\right)-\hbar^{0} \frac{1}{24} \theta_{0} .
$$

Note that $\left.\frac{1}{2} \sum_{k, l \geq 1}\left(p_{k+l} q_{k} q_{l}+p_{k} p_{l} q_{k+l}\right)\right)$ are the cut-and-join operators of $[\mathbf{7}]$. The full rubber potential can be related to Hurwitz numbers by use of a localization argument as in [18].

Example 3.10. As a consequence of Corollary 5.9, for $\left(\mathbb{P}^{1}, \mathcal{O}(1)\right)$, the terms in the rubber potential without powers of $\mathrm{L}^{\infty}$ corresponding to positive degree maps are

$$
\begin{aligned}
\hbar^{-1} & \frac{1}{2 \pi} \int_{0}^{2 \pi} \frac{\left(\beta_{0}+\sum_{k} p_{k, 0} e^{-i k x}+\sum_{k} q_{k, 0} e^{i k x}\right)^{2}}{2} \\
& \cdot\left(\beta_{2}+\sum_{k} p_{k, 2} e^{-i k x}+\sum_{k} q_{k, 2} e^{i k x}\right) d x \\
& +\hbar^{-1} \frac{1}{2 \pi} \int_{0}^{2 \pi} e^{\beta_{2}+\sum_{k} p_{k, 2} e^{-i k x}+\sum_{k} q_{k, 2} e^{i k x} z e^{i x} d x .}
\end{aligned}
$$

This follows from results in Section 5 .

3.3. Trivial cylinders. It was our convention to exclude trivial cylinders from the rubber potential. They will be accounted for by the algebra $\mathcal{R}$. To prove this, it will be advantageous to write down a potential including trivial cylinders and relate it to the rubber potential. Let $\Gamma$ be some rubber topological type. Let $\Gamma_{\mid}$be a rubber topological type obtained from $\Gamma$ by adjoining a degree $r$ trivial cylinder. From [11], we have

Theorem 3.11. There is a natural map

$$
v: \mathcal{M}\left(\mathcal{A}, \Gamma_{\mid}\right) \longrightarrow \mathcal{M}(\mathcal{A}, \Gamma) \times X
$$

so that

$$
v_{*}\left[\mathcal{M}\left(\mathcal{A}, \Gamma_{\mid}\right)\right]^{\mathrm{vir}}=\frac{1}{r}[\mathcal{M}(\mathcal{A}, \Gamma)]^{\mathrm{vir}} \times[X]
$$

and

$$
v^{*}\left(\mathrm{~L}^{\infty}\right)=\mathrm{L}^{\infty}
$$

Consequently, if $\Gamma$ has $m$ interior marked points and $r_{0}+r_{\infty}$ boundary marked points, then we have a commutative diagram

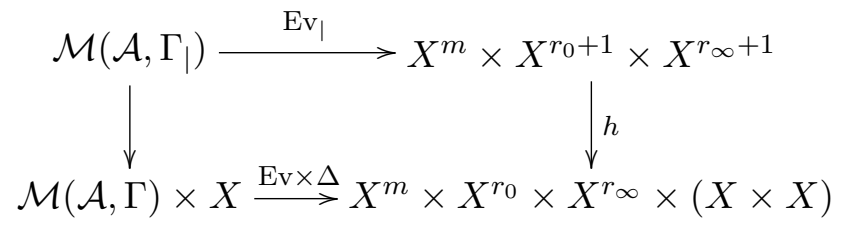


where $\Delta: X \rightarrow X^{2}$ is the diagonal and the morphism $h$ reorders the products of $X$ so that the product of $X$ s corresponding to the $\left(r_{0}+1\right)$-st and $\left(r_{\infty}+1\right)$-st boundary marked points are taken to $X \times X$.

For classes $c \in H^{*}\left(X^{m} \times X^{r_{0}+1} \times X^{r_{\infty}+1}\right)$, we have

$$
\begin{aligned}
& \operatorname{deg}\left(\operatorname{Ev}_{\mid}^{*}(c) \cap\left[\mathcal{M}\left(\mathcal{A}, \Gamma_{\mid}\right)\right]^{\operatorname{vir}}\right) \\
& \quad=\frac{1}{r} \operatorname{deg}\left(\left((\operatorname{Ev} \times \Delta) \circ h^{-1}\right)^{*}(c) \cap\left([\mathcal{M}(\mathcal{A}, \Gamma)]^{\operatorname{vir}} \times[X]\right)\right) .
\end{aligned}
$$

Definition 3.12. The rubber potential with trivial cylinders, $A_{\mid}$is defined as before except that we allow the set $\Xi$ to contain topological types that have trivial cylinders as components.

Define the action of an algebra of power series in infinitely many noncommuting variables $\kappa_{1}, \kappa_{2}, \ldots$ on monomials $f \in \mathcal{R}$ by

$$
\kappa_{n} \cdot f=\frac{1}{n} \hbar^{-1} \sum_{i_{1}, i_{2}}(-1)^{\left(\operatorname{deg}_{p q}(f)\right)\left(\operatorname{deg}\left(p_{n, i_{1}}\right)\right)} g^{i_{1} i_{2}} p_{n, i_{1}} f q_{n, i_{2}},
$$

where $\operatorname{deg}_{p q}(f)$ is the sum of the degrees of the $p$ and $q$ variables in $f$. Extend the action linearly to $\mathcal{R}$. Let

$$
T K=\sum_{n} \kappa_{n}
$$

and define a map

$$
\begin{aligned}
& T: \mathcal{R} \longrightarrow \mathcal{R} \\
& T: f \longmapsto e^{T K} f .
\end{aligned}
$$

Lemma 3.13. $T$ takes the rubber potential to the rubber potential with trivial cylinders,

$$
T(A)=A_{\mid} .
$$

Proof. The proof is straightforward. The factorial terms in the exponential come from relabeling the boundary marked points.

Definition 3.14. Let $f$ and $h$ be elements in $\mathcal{R}$. We define a binary operation $f * \mid h$ as follows. Introduce a set of auxiliary variables $\tilde{p}_{n, i}$, $\tilde{q}_{n, i}=\sum_{j} n \hbar g^{i j} \partial / \partial \tilde{p}_{n, j}$. Define

$$
f *|h=f(p, \tilde{q}) h(\tilde{p}, q)|_{\tilde{p}=0} .
$$

Note that in the above, we treat $\partial / \partial \tilde{p}_{n, j}$ as an element with the same parity as $p_{n, j}$. The operation $* \mid$ is the one that corresponds to stacking curves to form split maps. This will be elaborated in the section on degenerations.

Lemma 3.15. $T$ is a homomorphism from $(\mathcal{R}, *)$ to $\left(\mathcal{R}, *_{\mid}\right)$. 
Proof.

$$
\frac{\partial}{\partial p_{n, i}}\left(e^{T K} 1\right)=\hbar^{-1} \frac{1}{n} \sum_{j}\left(e^{T K} 1\right) g^{i j} q_{n, j}
$$

which implies for $f$, a monomial,

$$
\frac{\partial}{\partial p_{n, i}}\left(e^{T K} f\right)=\hbar^{-1} \frac{1}{n} \sum_{j}(-1)^{\left(\operatorname{deg}_{p q} f\right)\left(\operatorname{deg} p_{n, i}\right)}\left(e^{T K} f\right) g^{i j} q_{n, j}+e^{T K} \frac{\partial f}{\partial p_{n, i}} .
$$

The lemma follows by induction on the number of $p$ and $q$ variables in $f$.

Definition 3.16. The rational potential $A$ is

$$
A_{\text {rat }}=\sum_{d \in B_{1}(X)}(\Gamma, P, Q)_{g=0, d, m}^{\bullet} z^{d}
$$

where the sum is taken over moduli spaces $\mathcal{M A}$ involving only connected domains of genus 0 .

\subsection{Action of $\mathcal{R}$ on $\mathcal{F}$.}

Lemma 3.17. $\mathcal{F}$ can be given the structure of a graded $\mathcal{R}$-module.

Proof. Consider the inclusion

$$
I: D \longrightarrow Z
$$

and the induced maps

$$
\begin{gathered}
I^{*}: H^{*}(Z) \longrightarrow H(D), \\
I^{* \vee}: H^{*}(D)^{\vee} \longrightarrow H^{*}(Z)^{\vee} .
\end{gathered}
$$

We define the action of $\mathcal{R}$ on $f \in \mathcal{F}$ as follows:

$$
\begin{aligned}
\lambda \cdot f & =0 \\
\hbar \cdot f & =\tilde{\hbar} f \\
p_{n, i} \cdot f & =\tilde{p}_{n, i} \\
q_{n, i} \cdot f & =\tilde{\hbar} n \sum_{i^{\prime}} g^{i i^{\prime}} \frac{\partial}{\partial \tilde{p}_{n, i^{\prime}}} f \\
\beta_{i} \cdot f & =I^{* \vee}\left(\beta_{i}\right) f \\
z^{d} \cdot f & =\tilde{z}^{I_{*} d} f,
\end{aligned}
$$

where $g^{i i^{\prime}}$ is the intersection pairing on $H^{*}(D)$.

Because

$$
\begin{aligned}
\operatorname{deg} \tilde{z}^{I_{*} d} & =\left\langle c_{1}(T Z), I_{*} d\right\rangle=\left\langle I^{*} c_{1}(T Z), d\right\rangle \\
& =\left\langle c_{1}(T D)+c_{1}(N), d\right\rangle=\operatorname{deg} z^{d},
\end{aligned}
$$

the action preserves grading. 
Definition 3.18. There is a bilinear operation

$$
\cdot_{l}: \mathcal{R} \otimes \mathcal{F} \longrightarrow \mathcal{F}
$$

Given a monomial

$$
h=\hbar^{-\frac{1}{2} \chi} \lambda^{l} \beta_{i_{1}} \cdots \beta_{i_{m}} p_{s_{1}^{0}, j_{1}} \cdots p_{s_{r_{0}}^{0}, j_{r_{0}}} q_{s_{1}^{\infty}, k_{1}} \cdots q_{s_{r_{\infty}}^{\infty}, k_{r_{\infty}}} z^{d} \in \mathcal{R}
$$

and $f \in \mathcal{F}$ we will define $h \cdot \mid f$ as follows: write

$$
q_{n, i}=\left(\sum_{i^{\prime}} g^{i i^{\prime}} \frac{\partial}{\partial \tilde{p}_{n, i^{\prime}}}\right),
$$

and define

$$
\begin{aligned}
h \cdot \mid f= & \left(\left(\tilde{\hbar}^{-\frac{1}{2} \chi} \delta_{l 0} I^{\vee}\left(\beta_{i_{1}}\right) \ldots I^{\vee}\left(\beta_{i_{m}}\right) p_{s_{1}^{0}, j_{1}} \ldots p_{s_{r_{0}}^{0}, j_{r_{0}}} q_{s_{1}^{\infty}, k_{1}} \ldots\right.\right. \\
& \left.\left.q_{s_{r_{\infty}}^{\infty}, k_{r_{\infty}}}\right) f\right)\left.\right|_{\tilde{p}=0},
\end{aligned}
$$

followed by substituting $\tilde{p}_{n, j}$ for $p_{n, j}$.

This operation corresponds to joining a curve in $\mathcal{M A}$ to one in $\mathcal{M Z}$. Analogously to the multiplication in $\mathcal{R}, \cdot \mid$ and $\cdot$ are related as follows.

Lemma 3.19. For $h \in \mathcal{R}, f \in \mathcal{F}$, we have

$$
T(h) \cdot f=h \cdot f .
$$

\section{Degeneration formulae}

Theorem 2.38 gives formulae relating the line-bundles

$$
\text { Dil, Split, } \mathrm{L}_{i, \text { not top }}, \mathrm{L}_{i, \text { not bot }}
$$

on $\mathcal{M}\left(\mathcal{Z}, \Gamma_{Z}\right)$ and $\mathcal{M}\left(\mathcal{A}, \Gamma_{A}\right)$. In this section, we will show that the first Chern classes of these line-bundles represent specific geometric situations involving split maps. For example, $c_{1}$ (Split) is dual to a substack of $\mathcal{M}\left(\mathcal{A}, \Gamma_{A}\right)$ made up of, in a virtual sense, all split maps. $c_{1}\left(\mathrm{~L}_{i, \text { not top }}\right)$ virtually consists of all split maps in which the $i$ th marked point is not on the top component. This allows us to write the cap product of a first Chern class of one of our bundles with the virtual cycle in terms of the virtual cycles of smaller moduli spaces. This provides degeneration formulae that can be expressed in the language of generating functions.

We will express the first Chern class of various line-bundles geometrically by adapting Li's argument [16]. The argument is in several stages, and we 
state it only in the case $\mathcal{M}(\mathcal{Z}, \Gamma)$.

(1) For $\Gamma$, consider quadruples $\Upsilon=\left(\Gamma_{A}, \Gamma_{Z}, L, J\right)$ so that the type join, $\Gamma_{A} *_{L, J} \Gamma_{Z}$ is isomorphic to $\Gamma$. We define a line-bundle $L_{\Upsilon}$ on $\mathcal{M}\left(\mathcal{Z}, \Gamma_{Z}\right)$.

(2) We show that

$$
c_{1}\left(L_{\Upsilon}\right) \cap[\mathcal{M}(\mathcal{Z}, \Gamma)]^{\mathrm{vir}}=m(\Upsilon)[\mathcal{M}(\mathcal{Z} \sqcup \mathcal{A}, \Upsilon)]^{\mathrm{vir}},
$$

where $[\mathcal{M}(\mathcal{Z} \sqcup \mathcal{A}, \Upsilon)]^{\text {vir }}$ is an appropriately defined virtual cycle. Therefore, $L_{\Upsilon}$ can be thought of as $\mathcal{O}(m(\Upsilon) \mathcal{M}(\mathcal{Z} \sqcup \mathcal{A}, \Upsilon))$.

(3) Given the joining morphism

$$
\Phi: \mathcal{M}\left(\mathcal{A}, \Gamma_{A}\right) \times_{D^{r}} \mathcal{M}\left(\mathcal{Z}, \Gamma_{Z}\right) \longrightarrow \mathcal{M}\left(\mathcal{Z}, \Gamma_{A} \sqcup_{L, J} \Gamma_{Z}\right)
$$

and the diagram

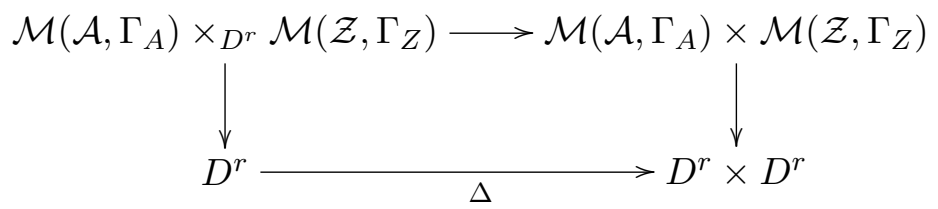

where $\Delta$ is the diagonal map, we have

$$
\Phi_{*} \Delta^{!}\left(\left[\mathcal{M}\left(\mathcal{A}, \Gamma_{A}\right)\right]^{\mathrm{vir}} \times\left[\mathcal{M}\left(\mathcal{Z}, \Gamma_{Z}\right)\right]^{\mathrm{vir}}\right)=[\mathcal{M}(\mathcal{A} \sqcup \mathcal{Z}, \Upsilon)]^{\mathrm{vir}} .
$$

(4) Given a line-bundle

$$
L=\text { Dil or } \mathrm{L}_{i, \text { ext }},
$$

we exhibit a set of join-equivalence classes $\Omega$ so that

$$
L=\otimes_{[\Upsilon] \in \Omega} L_{\Upsilon} .
$$

(5) Consequently,

$$
c_{1}(L) \cap[\mathcal{M}(\mathcal{Z}, \Gamma)]^{\mathrm{vir}}=\sum_{\Upsilon \in \Omega} m(\Upsilon) \Phi_{*} \Delta^{!}\left(\left[\mathcal{M}\left(\mathcal{A}, \Gamma_{A}\right)\right]^{\mathrm{vir}} \times\left[\mathcal{M}\left(\mathcal{Z}, \Gamma_{Z}\right)\right]^{\mathrm{vir}}\right) .
$$

To modify this argument to work for $\mathcal{M}(\mathcal{A}, \Gamma)$ replace all pairs $\left(\Gamma_{A}, \Gamma_{Z}\right)$ with $\left(\Gamma_{A_{t}}, \Gamma_{A_{b}}\right)$, replace $\mathcal{Z}$ with $\mathcal{A}$, and use the line-bundles

$$
L=\text { Split, } \mathrm{L}_{i, \text { not top }}, \mathrm{L}_{i, \text { not bot }} \text {. }
$$

4.1. Interpretation of bundles. Let us rewrite the bundles

$$
\text { Dil, } \mathrm{L}_{i, \text { ext }}, \mathrm{L}_{i, \text { not top }}, \mathrm{L}_{i, \text { not bot }}
$$

as tensor products of $L_{\Upsilon \mathrm{s}}$ on $\mathcal{M}(\mathcal{Z}, \Gamma)$ and $\mathcal{M}(\mathcal{A}, \Gamma)$.

On $\mathcal{M Z}$ where $i$ is the label for an interior marked point,

(1) $\Omega_{\text {Dil }}=\left\{\left(\Gamma_{A}, \Gamma_{Z}, L, J\right)\right\}$ the set of all join-equivalence classes of quadruples $\left(\Gamma_{A}, \Gamma_{Z}, L, J\right)$, 
(2) $\Omega_{\mathrm{L}_{i, \mathrm{ext}}}=\left\{\left(\Gamma_{A}, \Gamma_{Z}, L, J\right) \mid i \in J\left(M_{A}\right)\right\}$,

while on $\mathcal{M A}$ where $i, j$ are labels for interior marked points,

(1) $\Omega_{\text {Split }}=\left\{\left(\Gamma_{A_{t}}, \Gamma_{A_{b}}, L, J\right)\right\}$.

(2) $\Omega_{\mathrm{L}_{i, \text { not bot }}}=\left\{\left(\Gamma_{A_{t}}, \Gamma_{A_{b}}, L, J\right) \mid i \in J\left(M_{A_{t}}\right)\right\}$,

(3) $\Omega_{\mathrm{L}_{i, \text { not top }}}=\left\{\left(\Gamma_{A_{t}}, \Gamma_{A_{b}}, L, J\right) \mid i \in J\left(M_{A_{b}}\right)\right\}$,

(4) $\Omega_{(i, j)}=\left\{\left(\Gamma_{A_{t}}, \Gamma_{A_{b}}, L, J\right) \mid i \in J\left(M_{A_{t}}\right), j \in J\left(M_{A_{b}}\right)\right\}$,

(5) $\Omega_{(i j,)}=\left\{\left(\Gamma_{A_{t}}, \Gamma_{A_{b}}, L, J\right) \mid i, j \in J\left(M_{A_{t}}\right)\right\}$,

(6) $\Omega_{(, i j)}=\left\{\left(\Gamma_{A_{t}}, \Gamma_{A_{b}}, L, J\right) \mid i, j \in J\left(M_{A_{b}}\right)\right\}$.

Theorem 4.1 ([11]). For $L=\mathrm{Dil}_{,} \mathrm{L}_{i, \text { ext }}$, Split, $\mathrm{L}_{i, \text { not bot }}, \mathrm{L}_{i, \text { not top }}$,

$$
L=\bigotimes_{[\Upsilon] \in \Omega_{L}} L \Upsilon
$$

where $[\Upsilon]$ denotes a join-equivalence class and $\Upsilon$, a representative element.

4.2. Splitting of moduli stacks. We need to cite a number of results from [16]. These results were proved for a different moduli stack, $\mathcal{M}(\mathcal{W})$, but because of the explicit parallels between that space and $\mathcal{M}(\mathcal{A}, \Gamma)$ and $\mathcal{M}(\mathcal{Z}, \Gamma)$, the proofs can be modified in a straightforward fashion. We begin by relating the virtual cycle $[\mathcal{M}(\mathcal{A} \sqcup \mathcal{Z}, \Upsilon)]^{\text {vir }}$ defined in $[\mathbf{1 6}]$, where $\Upsilon=\left(\Gamma_{A}, \Gamma_{Z}, L, J\right)$ is a type join quadruple to other virtual cycles.

Theorem 4.2. We have the following equality among cycle classes

$$
c_{1}\left(L_{\Upsilon}\right) \cap\left[\mathcal{M}\left(\mathcal{Z}, \Gamma_{A} *_{L, J} \Gamma_{Z}\right)\right]^{\mathrm{vir}}=m(\Upsilon)[\mathcal{M}(\mathcal{A} \sqcup \mathcal{Z}, \Upsilon)]^{\mathrm{vir}}
$$

where $m(\Upsilon)$ is as in Definition 2.32.

Consider the fiber square

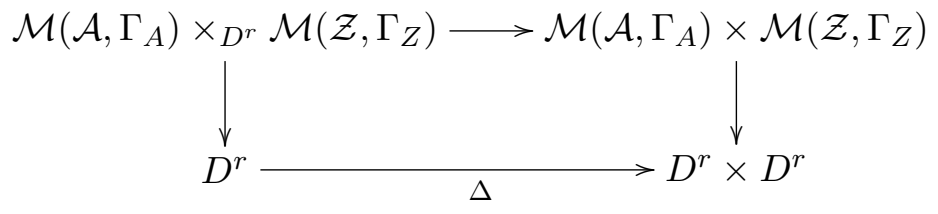

where $\Delta$ is the diagonal morphism and the downward maps are induced from evaluation maps at the boundary marked points of $\mathcal{M Z}$ and at the boundary marked points of $\mathcal{M A}$ mapping to $D_{\infty}$. Let the virtual cycle on the fiber product be given by

$$
\left[\mathcal{M}\left(\mathcal{A}, \Gamma_{A}\right) \times{ }_{D^{r}} \mathcal{M}\left(\mathcal{Z}, \Gamma_{Z}\right)\right]^{\mathrm{vir}}=\Delta^{!}\left(\left[\mathcal{M}\left(\mathcal{A}, \Gamma_{A}\right)\right]^{\mathrm{vir}} \times\left[\mathcal{M}\left(\mathcal{Z}, \Gamma_{Z}\right)\right]^{\mathrm{vir}}\right) .
$$

Theorem 4.3. If

$$
M_{[\Upsilon]}=\coprod_{\left(\Gamma_{A}^{\prime}, \Gamma_{Z}^{\prime}, L, J\right) \in[\Upsilon]} \mathcal{M}\left(\mathcal{A}, \Gamma_{A}^{\prime}\right) \times_{D^{r}} \mathcal{M}\left(\mathcal{Z}, \Gamma_{Z}^{\prime}\right)
$$

is given the virtual cycle of a disjoint union, then

$$
\Phi_{[\Upsilon]}: M_{[\Upsilon]} \longrightarrow \mathcal{M}\left(\mathcal{Z}, \Gamma_{A} *_{L, J} \Gamma_{Z}\right)
$$


satisfies

$$
\Phi_{[\Upsilon] *}\left([M]^{\mathrm{vir}}\right)=\left|M_{Z}\right| !\left|M_{A}\right| !\left(\left|R_{Z}\right| !\right)^{2}[\mathcal{M}(\mathcal{A} \sqcup \mathcal{Z}, \Upsilon)]^{\mathrm{vir}} .
$$

Note that the multiplicity term is natural in light of Proposition 2.34.

\section{Corollary 4.4.}

$$
c_{1}\left(L_{\Upsilon}\right) \cap\left[\mathcal{M}\left(\mathcal{Z}, \Gamma_{A} *_{L, J} \Gamma_{Z}\right)\right]^{\mathrm{vir}}=\frac{m(\Upsilon)}{\left|M_{Z}\right| !\left|M_{A}\right| !\left(\left|R_{Z}\right| !\right)^{2}} \Delta^{!}\left(M_{\Upsilon}\right) .
$$

$L$ together with $I: X \rightarrow Z$ induces a morphism

$$
\Lambda:\left(X^{\left|M_{A}\right|} \times X^{\left|R_{A 0}\right|}\right) \times Z^{\left|M_{Z}\right|} \longrightarrow Z^{M} \times X^{R},
$$

where $M=\left|M_{Z}\right|+\left|M_{A}\right|$ and $R=\left|R_{A 0}\right|$ are the number of interior and boundary marked points in $\Gamma_{A} *_{L, J} \Gamma_{Z}$.

We have morphisms

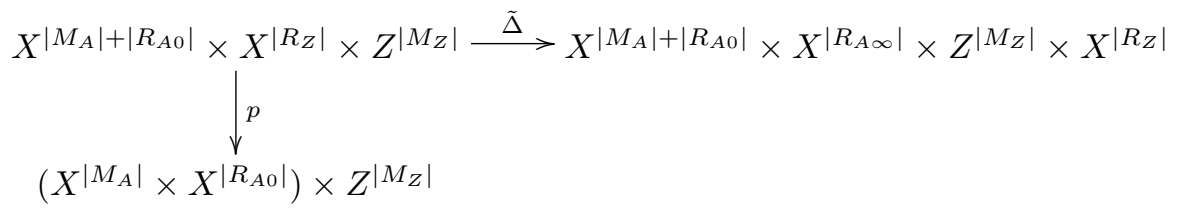

where $\tilde{\Delta}$ is induced by $\Delta: X^{\left|R_{Z}\right|} \rightarrow X^{\left|R_{A \infty}\right|} \times X^{\left|R_{Z}\right|}$ and $p$ is the projection.

Therefore, for $c \in H^{*}\left(Z^{\left|M_{Z}\right|} \times X^{\left|R_{Z}\right|}\right.$,

$$
\begin{gathered}
\operatorname{deg}\left(\left(\operatorname{Ev}^{*}(c) \cup c_{1}\left(L_{\Upsilon}\right)\right) \cap\left[\mathcal{M}\left(\mathcal{Z}, \Gamma_{A}{ }_{L, J} \Gamma_{Z}\right)\right]^{\mathrm{vir}}\right) \\
=\frac{m(\Upsilon)}{\operatorname{Aut}} \operatorname{deg}\left(\operatorname{Ev}^{*}\left(\tilde{\Delta}_{!}\left(p^{*} \Lambda^{*} c\right)\right) \cap\left(\left[\mathcal{M}\left(\mathcal{A}, \Gamma_{A}\right)\right]^{\mathrm{vir}} \times\left[\mathcal{M}\left(\mathcal{Z}, \Gamma_{Z}\right)\right]^{\mathrm{vir}}\right)\right)
\end{gathered}
$$

where Aut $=\operatorname{Aut}_{\Gamma_{A}, \Gamma_{Z}, L}\left(R_{A \infty}, R_{Z}\right)$.

If $\Omega$ is one of the sets of join-equivalence classes, from

$$
c_{1}\left(L_{\Omega}\right)=\sum_{[\Upsilon] \in \Omega} c_{1}\left(L_{\Upsilon}\right)
$$

we have

\section{Theorem 4.5.}

$$
\begin{aligned}
& \operatorname{deg}\left(\left(\operatorname{Ev}^{*}(c) \cup c_{1}\left(L_{\Omega}\right)\right) \cap[\mathcal{M}(\mathcal{Z}, \Gamma)]^{\mathrm{vir}}\right) \\
& \quad=\sum_{[\Upsilon] \in \Omega} \frac{m(\Upsilon)}{\left|M_{Z}\right| !\left|M_{A}\right| !\left|R_{Z}\right| !^{2}} \operatorname{deg}\left(\operatorname{Ev}^{*}\left(\tilde{\Delta}_{!}\left(p^{*} \Lambda^{*} c\right)\right) \cap\left[M_{[\Upsilon]}\right]^{\mathrm{vir}}\right)
\end{aligned}
$$

and analogously for $\mathcal{M A}$. 
Definition 4.6. Let the symbols

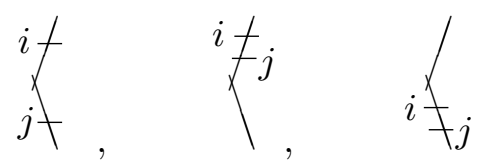

denote the cohomology classes

$$
\sum_{[\Upsilon] \in \Omega_{(i, j)}} c_{1}\left(L_{\Upsilon}\right), \quad \sum_{[\Upsilon] \in \Omega_{(i j,)}} c_{1}\left(L_{\Upsilon}\right), \quad \sum_{[\Upsilon] \in \Omega_{(, i j)}} c_{1}\left(L_{\Upsilon}\right),
$$

respectively.

These cohomology classes are dual to the cycles in $\mathcal{M A}$ representing split maps with $i$ and $j$ on top or bottom components as specified in the symbol, counted with the appropriate weight.

4.3. Normal bundle to split maps. The following is useful for localization computations.

Let $\left(\Gamma_{A}, \Gamma_{Z}, L, J\right)$ be type join quadruple. Consider $L_{\Upsilon}$ for the quadruple $\Upsilon=\left(\Gamma_{A}, \Gamma_{Z}, L, J\right)$. Then $c_{1}\left(L_{\Upsilon}\right)$ is a substack of $\mathcal{M}\left(\mathcal{Z}, \Gamma_{A} *_{L, J} \Gamma_{Z}\right)$.

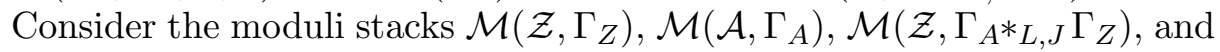
the inclusion

$$
\Phi: \mathcal{M}\left(\mathcal{A}, \Gamma_{A}\right) \times_{D^{r}} \mathcal{M}\left(\mathcal{Z}, \Gamma_{Z}\right) \longrightarrow \mathcal{M}\left(\mathcal{Z}, \Gamma_{A} *_{L, J} \Gamma_{Z}\right) .
$$

$\mathcal{M}\left(\mathcal{A}, \Gamma_{A}\right) \times{ }_{D^{r}} \mathcal{M}\left(\mathcal{Z}, \Gamma_{Z}\right)$ has projections $p_{A}, p_{Z}$ to its $\mathcal{M A}$ and $\mathcal{M Z}$ factors.

Theorem 4.7. On $\mathcal{M}\left(\mathcal{Z}, \Gamma_{A}{ }^{*}{ }_{L, J} \Gamma_{Z}\right), \Phi^{*} L_{\Upsilon}=p_{Z}^{*} \operatorname{Dil}^{\vee} \otimes p_{A}^{*} \mathrm{~L}^{\infty \vee}$.

Similarly, we have

Theorem 4.8. On $\mathcal{M}\left(\mathcal{A}, \Gamma_{A_{t}}{ }_{L, J} \Gamma_{A_{b}}\right), \Phi^{*} L_{\Upsilon}=p_{A_{b}}^{*} \mathrm{~L}^{0^{\vee}} \otimes p_{A_{t}}^{*} \mathrm{~L}^{\infty \vee}$.

4.4. Degeneration formulae. The above degeneration formulae can be written in terms of generating functions.

For $L=$ Dil, we can write down a potential $F_{\text {Dil }}$ which is defined by a formula similar to that of the relative potential except that instead of evaluating all possible cohomology classes on $[\mathcal{M Z}]^{\text {vir }}$, we evaluate them on $c_{1}(\mathrm{Dil}) \cap[\mathcal{M Z}]^{\text {vir }}$. That is, we define the Dil-correlator by for $a_{1}, \ldots, a_{n} \in$ $H^{*}(Z), b_{1}, \ldots, b_{r} \in H^{*}(X)$

$$
\begin{aligned}
& \left\langle a_{1} \ldots a_{m}, b_{1} \ldots b_{r}\right\rangle_{\operatorname{Dil}, \chi, A,\left(s_{1}, \ldots, s_{r}\right)} \\
& \quad=\sum_{\Gamma \in \Xi}\left(\operatorname{Ev}^{*}\left(a_{1} \times \cdots \times a_{m} \times b_{1} \times \cdots \times b_{r}\right) \cup c_{1}(\operatorname{Dil})\right) \cap[\mathcal{M}(\mathcal{Z}, \Gamma)]^{\mathrm{vir}}
\end{aligned}
$$

and define $F_{\text {Dil }}$ as in the previous section. Define the rubber potential with trivial cylinders without powers of $c_{1}\left(\mathrm{~L}^{\infty}\right)$ by

$$
A_{\left.\right|_{\lambda=0}}=\left(A_{\mid}\right)_{\lambda=0}
$$


Then, by Lemma 3.19, Theorems 4.1, and 4.5, we have

\section{Theorem 4.9.}

$$
\begin{aligned}
& F_{\text {Dil }}=A_{\left.\right|_{\lambda=0} \cdot \mid} \cdot F \\
& F_{\text {Dil }}=A_{\lambda=0} \cdot F .
\end{aligned}
$$

To study insertions of $c_{1}\left(\mathrm{~L}_{i, \text { ext }}\right)$, we choose an element $e_{j}$ of our basis for $H^{*}(Z)$. Because $c_{1}\left(\mathrm{~L}_{i, \text { ext }}\right)$ is dependent on the choice of marked point, we add a distinguished marked point to all of the relative topological types that contribute to our potential. At this marked point, we evaluate $c_{1}\left(\mathrm{~L}_{i \text {,ext }}\right) \cup$ $\mathrm{ev}_{i}^{*} e_{j}$. More formally, given a relative topological type $\Gamma$ with $m$ marked points, consider the set $D(\Gamma)$ consisting of all relative topological types $\Gamma^{\prime}$ with $(m+1)$ marked points such that when we forget $(m+1)$-st marked point on $\Gamma^{\prime}$, we obtain $\Gamma$. Define the extended component correlator for $a_{1}, \ldots, a_{m} \in$ $H^{*}(Z), b_{1}, \ldots, b_{r} \in H^{*}(X=D)$ by

$$
\begin{aligned}
& \left\langle a_{1} \cdots a_{m}, c_{1} \cdots c_{r}\right\rangle_{\left(\mathrm{ex}, e_{j}\right), \chi, d,\left(s_{1}, \cdots, s_{r}\right)} \\
& =\sum_{(\Gamma, k) \in \Xi}\left(\sum _ { \Gamma ^ { \prime } \in D ( \Gamma ) } \left(\operatorname{Ev}^{*}\left(a_{1} \times \cdots \times a_{m} \times e_{j} \times b_{1} \times \cdots \times b_{r}\right)\right.\right. \\
& \left.\left.\quad \cup c_{1}\left(\mathrm{~L}_{m+1, \mathrm{ext}}\right) \cap\left[\mathcal{M}\left(\mathcal{Z}, \Gamma^{\prime}\right)\right]^{\mathrm{vir}}\right)\right) .
\end{aligned}
$$

Define extended component generating function $F_{\mathrm{ex}, j}$ by substituting the extended component correlator into the definition of the relative potential. Then, for $I: X=D \rightarrow Z$, we write

$$
I^{*} e_{j}=\sum_{l} M_{j l} c_{l}
$$

for $M_{j l} \in \mathbb{Q}$ which gives

$$
\begin{aligned}
F_{\mathrm{ex}, j} & =\sum_{l} M_{j l} \frac{\partial\left(A_{\mid}\right)_{\lambda=0}}{\partial \beta_{l}} \cdot{ }_{\mid} F \\
& =\sum_{l} M_{j l} \frac{\partial A_{\lambda=0}}{\partial \beta_{l}} \cdot F .
\end{aligned}
$$

Now, since $\mathrm{L}_{k, \mathrm{ext}}=\mathrm{ev}_{k}^{*}([D])$, we have

Theorem 4.10. Let $e_{j}$ be an element of our basis for $H^{*}(Z)$. Let $N$ be a matrix defined by

$$
e_{j} \cup[D]=\sum_{l} N_{j l} e_{l}
$$

then

$$
\sum_{l} N_{j l} \frac{\partial F}{\partial \theta_{l}}=\sum_{l} M_{j l} \frac{\partial A_{\lambda=0}}{\partial \beta_{l}} \cdot F
$$


Proof. $\mathrm{ev}_{k}^{*} e_{j} \cup c_{1}\left(\mathrm{~L}_{k, \text { ext }}\right)=\left(\operatorname{ev}_{k}^{*}\left(e_{j} \cup[D]\right)\right)$ implies

$$
F_{\text {ex }, j}=\sum_{l} N_{j l} \frac{\partial F}{\partial \theta_{l}} \text {. }
$$

Likewise, we can write down a rubber potential with $c_{1}$ (Split) inserted in the correlator. This is analogous to Dil in the relative case.

Theorem 4.11. $A_{\text {Split }}=A_{\lambda=0} * A$.

We can also write down a potential involving insertions of $c_{1}\left(\mathrm{~L}_{i, \text { not bot }}\right)$. This is analogous to $\mathrm{L}_{i \text {,ext }}$ in the relative case. Again, we have to single out a cohomology class $c_{j} \in H^{*}(X)$ where at some marked point $i$, we will evaluate $\operatorname{ev}_{i}^{*}\left(c_{j}\right)$ and $\mathrm{L}_{i, \text { not bot }}$.

Theorem 4.12. $A_{\mathrm{L}_{i, \text { not bot }, c_{j}}}=\frac{\partial A_{\lambda=0}}{\partial \beta_{j}} * A$.

Similarly, for $\mathrm{L}_{i, \text { not top }}$, we have

Theorem 4.13. $A_{\mathrm{L}_{i, \mathrm{not} \text { top }, c_{j}}}=A_{\lambda=0} * \frac{\partial A}{\partial \beta_{j}}$.

From Theorem 2.38(3) one can obtain a degeneration formula for the rubber potential by inserting

$$
c_{1}\left(\mathrm{~L}^{\infty} \otimes \operatorname{ev}_{i}^{*} L\right) \operatorname{ev}_{i}^{*}\left(c_{j}\right)=\left(c_{1}\left(\mathrm{~L}^{\infty}\right)+e v_{i}^{*} c_{1}(L)\right) \operatorname{ev}_{i}^{*}\left(c_{j}\right)
$$

at a distinguished point in the rubber potential to compute $A_{\mathrm{L}_{i, \text { not top }}, c_{j}}$. Note that $\frac{\partial A}{\partial \lambda}$ is the rubber potential with an extra insertion of $c_{1}\left(\mathrm{~L}^{\infty}\right)$.

Theorem 4.14. Define the matrix $N_{i j}$ by

$$
c_{1}(L) \cup c_{i}=\sum_{j} N_{i j} c_{j} .
$$

Then for each $i$, we have

$$
\frac{\partial}{\partial \lambda} \frac{\partial A}{\partial \beta_{i}}+\sum_{j} N_{i j} \frac{\partial A}{\partial \beta_{j}}=\frac{\partial A_{\lambda=0}}{\partial \beta_{i}} * A .
$$

4.5. Reconstruction of the relative potential. Let us look at the relative case with $(Z, D)$. Let $L$ be the normal bundle to $D$ in $Z$. We can use the degeneration formula for $\mathrm{L}_{i \text {,ext }}$ to reconstruct the relative Gromov-Witten invariants of $(Z, D)$ from the rubber potential (without powers of $\mathrm{L}^{\infty}$ ) of $(D, L)$ and from seed values of the relative Gromov-Witten invariants of $(Z, D)$. This is a formal consequence of the fact that $\mathrm{ev}_{i}^{*} D=\mathrm{L}_{i \text {,ext }}$ and Theorem 4.10.

We need to choose a particular basis for $H^{*}(Z)$. Let $V \subseteq H^{*}(Z)$ be the subspace

$$
V=\operatorname{im}\left(\cup[D]: H^{*-2}(Z) \longrightarrow H^{*}(Z)\right) .
$$


Let $e_{1}, \ldots, e_{v}$ be a homogeneous basis for $V$, ordered by degree. Extend this to a basis $\left\{e_{v+1}, \ldots, e_{v+w}\right\}$ of $H^{*}(Z)$.

Theorem 4.15. The relative potential $F$ of $(Z, D)$ can be reconstructed from the rubber potential of $(D, N)$ together with the relative potential involving only the classes $\left\{e_{v+1}, \ldots, e_{v+w}\right\}$, that is, from

$$
\left.F\right|_{\theta_{1}=\theta_{2}=\cdots=\theta_{v}=0} .
$$

Proof. We add in one $\theta_{i}$ variable at a time. So, suppose we have determined

$$
\left.F\right|_{\theta_{j}=\cdots=\theta_{v}=0} .
$$

Since $e_{j} \in V$,

$$
e_{j}=\sum_{k} a_{k} e_{k} \cup[D]
$$

for $a_{k} \in \mathbb{Q}$ where $a_{l}=0$ for $l \in\{j, \ldots, v\}$ for degree reasons. By the above, we have

$$
\frac{\partial F}{\partial \theta_{j}}=\sum_{k, l} a_{k} M_{k l} \frac{\partial A_{\lambda=0}}{\partial \beta_{l}} \cdot F
$$

which allows us to solve for

$$
\left.F\right|_{\theta_{j+1}=\cdots=\theta_{v}=0} .
$$

\subsection{Transferring classes between components of split maps.}

Theorem 4.16. On $\mathcal{M A}$ with two distinguished interior marked points, the following equation holds among divisors.

$$
\mathrm{ev}_{2}^{*}\left(c_{1}(L)\right)-\mathrm{ev}_{1}^{*}\left(c_{1}(L)\right)=f_{2 f}-2^{2 f} .
$$

Proof. Recall the following facts:

$$
\begin{aligned}
c_{1}\left(\mathrm{~L}^{0}\right)+c_{1}\left(\mathrm{~L}^{\infty}\right) & =c_{1}(\text { Split }) \\
c_{1}\left(\mathrm{~L}^{0}\right) & =c_{1}\left(\mathrm{~L}_{i, \text { not top }}\right)+\mathrm{ev}_{i}^{*} c_{1}(L) \\
c_{1}\left(\mathrm{~L}^{\infty}\right) & =c_{1}\left(\mathrm{~L}_{i, \text { not bot }}\right)-\mathrm{ev}_{i}^{*} c_{1}(L) .
\end{aligned}
$$


Putting these together, we see

$$
c_{1}(\text { Split })=c_{1}\left(\mathrm{~L}_{1, \text { not top }}\right)-\mathrm{ev}_{1}^{*} c(L)+c_{1}\left(\mathrm{~L}_{2, \text { not top }}\right)+\mathrm{ev}_{2}^{*} c_{1}(L) .
$$

Diagrammatically, this equation can be expressed as

$$
\begin{aligned}
& { }^{1 \neq}+2 f+2 f+1 f+1 f_{2} \\
& ={ }_{1 f}^{2 f}+{ }_{1} f_{2}-\mathrm{ev}_{1}^{*} c_{1}(L)+{ }_{1 f}^{2 f}+{ }^{1 \neq} f^{2}+\mathrm{ev}_{2}^{*} c_{1}(L) \text {. }
\end{aligned}
$$

The result follows.

One can use this result to reconstruct all rubber invariants from those where all powers of $c_{1}(L)$ are at a single interior marked point.

\section{Examples}

5.1. Computation of the rational rubber potential. We compute the rational rubber potential of $L=\mathcal{O}(n)$ over $\mathbb{P}^{r}$. We do this by comparing the rubber potential to the rational Gromov-Witten invariants of $\mathbb{P}^{r}$. There is a geometric reason why this should be possible. Given a smooth rational curve, $\mathbb{P}^{1}$ with interior and boundary marked points,

$$
\left\{x_{1}, \ldots, x_{m}, p_{1}^{0}, \ldots, p_{r_{0}}^{0}, p_{1}^{\infty}, \ldots, p_{r_{\infty}}^{\infty}\right\}
$$

with multiplicities $\left(s_{1}^{0}, \ldots, s_{r_{0}}^{0}\right),\left(s_{1}^{\infty}, \ldots, s_{r_{\infty}}^{\infty}\right)$, specifying a rubber map to $L$ is equivalent to finding a degree $d$ map of $f: \mathbb{P}^{1} \rightarrow \mathbb{P}^{r}$ and a nowhere zero section (defined up to $\mathbb{C}^{*}$-action) of

$$
f^{*} L \otimes \mathcal{O}\left(-s_{1}^{0} p_{1}^{0}-\cdots-s_{r_{0}}^{0} p_{r_{0}}^{0}+s_{1}^{\infty} p_{1}^{\infty}+\cdots+s_{r_{\infty}}^{\infty} p_{r_{\infty}}^{\infty}\right) .
$$

The numerical condition in Lemma 2.20 for multiplicities implies that this bundle must be trivial. Therefore, there is only one section up to multiplication by an element of $\mathbb{C}^{*}$. The automorphism group of the map to rubber is equal to that of the stable map, so the rubber invariant should equal the Gromov-Witten invariant.

Unfortunately, this intuitive picture may not be true for singular curves. One, however, is able to prove these results when the target is $\mathbb{P}^{r}$ in which case rubber invariants count maps of smooth curves.

Let $L=\mathcal{O}(n)$ be a line-bundle over $\mathbb{P}^{r}$. We consider a stack $\mathcal{M A}$ of rubber maps to $(X, L)$. 
To prove that intersections on $\mathcal{M A}$ occur away from singular curves, we will use a Kleiman-Bertini theorem argument. Consider the commutative diagram

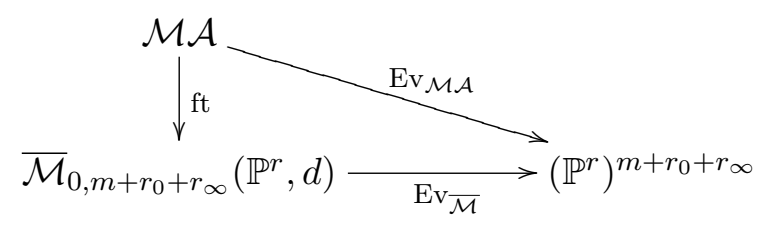

where $\mathrm{ft}$ is the map that takes a rubber map to $\mathbb{P}_{\mathbb{P}^{r}}(\mathcal{O}(n) \oplus \mathcal{O})$ to its projection to $\mathbb{P}^{r}$, contracts unstable components, and sees boundary and interior marked points as marked points.

We need the following well known:

Theorem 5.1. $\overline{\mathcal{M}}_{0, m}\left(\mathbb{P}^{r}, d\right)$ is non-singular and $\mathrm{ev}_{i}: \overline{\mathcal{M}}_{0, m}\left(\mathbb{P}^{r}, d\right) \rightarrow \mathbb{P}^{r}$ is a smooth morphism.

Theorem 5.2. The locus of maps with singular domain in $\overline{\mathcal{M}}_{0, m}\left(\mathbb{P}^{r}, d\right)$ is the finite union of substacks $M^{\prime}$ so that

(1) $M^{\prime}$ is non-singular and of positive codimension,

(2) The evaluation map Ev: $M^{\prime} \rightarrow X^{m}$ is smooth.

Now, we will use the Kleiman-Bertini theorem in the following form.

Theorem 5.3. Let $f: X \rightarrow\left(\mathbb{P}^{r}\right)^{p}$ be a morphism from $X$, a non-singular stack. Let $c=[H]^{a_{1}} \times \cdots \times[H]^{a_{p}} \in H^{l}\left(\left(\mathbb{P}^{r}\right)^{p}\right)$, where $[H]$ is a hyperplane class. Then there exists an open set $U \subseteq\left(\left(\mathbb{P}^{r}\right)^{\vee}\right)^{l}$, each point of which corresponds to a product of linear subspaces $K=V_{1} \times \cdots \times V_{p}$, Poincare-dual to $c$, such that $f^{-1}(K)$ is either empty or non-singular of dimension $\operatorname{dim} X-l$.

Let us first show that there is no contribution coming from rational curves with disconnected domain.

Theorem 5.4. Let $\mathcal{M A}$ be some rubber moduli space consisting of maps from the disjoint union of $q \geq 2$ rational curves, none of which are trivial cylinders. Let $l=\operatorname{vdim} \mathcal{M A}$. If $c \in H^{l}\left(\left(\mathbb{P}^{r}\right)^{m+r_{0}+r_{\infty}}\right)$, then there exists $K$, a product of linear subspaces in $\left(\mathbb{P}^{r}\right)^{m+r_{0}+r_{\infty}}$, Poincare-dual to $c$ so that $E v_{\mathcal{M A}}^{-1}(K)$ is empty and, therefore,

$$
\operatorname{deg}\left(\operatorname{Ev}_{\mathcal{M A}}^{*}(c) \cap[\mathcal{M} \mathcal{A}]^{\text {vir }}\right)=0 .
$$

Proof. Let us write $\overline{\mathcal{M}}=\overline{\mathcal{M}}_{\chi, m+r_{\infty}+r_{0}}^{\circ}\left(\mathbb{P}^{r}, d\right)$ for the moduli space of stable maps with domain the disjoint union of $q$ rational curves to $\mathbb{P}^{r}$ of degree $d$. $\chi=2 q$ is the Euler characteristic of the domain. Then, by the dimension formula,

$$
\operatorname{vdim} \mathcal{M} \mathcal{A}-\operatorname{vdim} \overline{\mathcal{M}}=\frac{1}{2} \chi-1=q-1>0 .
$$


Therefore, the codimension of the class $c \in H^{l}\left(\left(\mathbb{P}^{r}\right)^{m+r_{0}+r_{\infty}}\right)$ exceeds the dimension of $\overline{\mathcal{M}}$, so its Poincare-dual can be represented by a product of linear subspaces, $K$ so that that $\operatorname{Ev}_{\overline{\mathcal{M}}}(K)$ is empty.

Now, let us compute the rational rubber invariants coming from curves with connected domains using the following straightforward lemma.

Lemma 5.5. A map in $\mathcal{M A}$ with connected rational singular domain is mapped by ft to a map with singular domain in $\overline{\mathcal{M}}_{0, m+r_{0}+r_{\infty}}\left(\mathbb{P}^{r}, d\right)$.

Combining the above theorems and lemma, we get the following theorem.

Theorem 5.6. Let $\mathcal{M A}$ be some rubber moduli space with target $(X, L)$ and evaluation map

$$
\mathrm{Ev}: \mathcal{M A} \longrightarrow\left(\mathbb{P}^{r}\right)^{m} \times\left(\mathbb{P}^{r}\right)^{r_{0}} \times\left(\mathbb{P}^{r}\right)^{r_{\infty}}
$$

Let $l=\operatorname{vdim} \mathcal{M A}$. Then for $c \in H^{l}\left(\left(\mathbb{P}^{r}\right)^{m+r_{0}+r_{\infty}}\right)$, there exists a product of linear subspaces $K$, Poincare-dual to $c$ so that $\mathrm{Ev}^{-1}(K)$ is a finite union of reduced points, supported away from maps with singular domain.

Now, we specify the rubber moduli space we are considering. Let the genus be 0 . Fix a degree $d L \in H_{2}\left(\mathbb{P}^{r}\right)>0$, a number of interior marked points $m$ and boundary marked points with tangencies to $D_{0}$ and $D_{\infty}, r_{0}$ and $r_{\infty}$, respectively. Choose multiplicities $s_{1}^{0} \cdots s_{r_{0}}^{0}$ and $s_{1}^{\infty} \cdots s_{r_{\infty}}^{\infty}$ so that

$$
\left(s_{1}^{0}+\cdots+s_{r_{0}}^{0}\right)-\left(s_{1}^{\infty}+\cdots+s_{r_{\infty}}^{\infty}\right)=n d .
$$

Then, given a stable map $(f, C) \in \overline{\mathcal{M}}_{0, m+r_{0}+r_{\infty}}\left(\mathbb{P}^{r}, d\right)$ with smooth domain and marked points,

$$
\left\{x_{1}, \ldots, x_{m}, p_{1}^{0}, \ldots, p_{r_{0}}^{0}, p_{1}^{\infty}, \ldots, p_{r_{\infty}}^{\infty}\right\}
$$

the invertible sheaf

$$
\mathcal{L}=f^{*} L \otimes \mathcal{O}\left(-\left(s_{1}^{0} p_{1}^{0}+\cdots+s_{r_{0}}^{0} p_{r_{0}}^{0}\right)+\left(s_{1}^{\infty} p_{1}^{\infty}+\cdots+s_{r_{\infty}}^{\infty} p_{r_{\infty}}^{\infty}\right)\right)
$$

has degree $0 . \mathcal{L}$ s non-zero section, defined up to $\mathbb{C}^{*}$ induces a map

$$
C \longrightarrow \mathbb{P}_{\mathbb{P}^{r}}\left(L \oplus 1_{\mathbb{P}^{r}}\right)
$$

giving a point in $\mathcal{M A}$. The automorphisms of the map in $\overline{\mathcal{M}}_{0, m+r_{0}+r_{\infty}}\left(\mathbb{P}^{r}, d\right)$ are in bijective correspondence with the automorphisms of the map in $\mathcal{M A}$.

Theorem 5.7. With the data on $\mathcal{M A}$ as above,

$$
l=\operatorname{vim} \mathcal{M} \mathcal{A}, c \in H^{l}\left(\left(\mathbb{P}^{r}\right)^{m+r_{0}+r_{\infty}}\right),
$$

we have the following equality of rubber and Gromov-Witten invariants

$$
\operatorname{deg}\left(\operatorname{Ev}_{\mathcal{M}}^{*}(c) \cap \overline{\mathcal{M}}_{0, m+r_{0}+r_{\infty}}\left(\mathbb{P}^{r}, d\right)\right)=\operatorname{deg}\left(\operatorname{Ev}_{\mathcal{M} \mathcal{A}}^{*}(c) \cap[\mathcal{M A}]^{\mathrm{vir}}\right) .
$$


Proof. Choose a representative cycle $K$ as above. Then $\operatorname{Ev}_{\frac{-1}{\mathcal{M}}}(K)$ is a zerodimensional reduced substack of $\overline{\mathcal{M}}_{0, m+r_{0}+r_{\infty}}\left(\mathbb{P}^{r}, d\right)$ corresponding to maps with smooth domains. $\operatorname{Ev}_{\mathcal{M} \mathcal{A}}^{-1}(K)=\mathrm{ft}^{-1}\left(\operatorname{Ev}_{\overline{\mathcal{M}}}^{-1}(K)\right)$. By the above considerations, given an integral zero-dimensional substack, $x$ in $\operatorname{Ev}_{\overline{\mathcal{M}}}^{-1}(K), \mathrm{ft}^{-1}(x)$ is an integral zero-dimensional substack with the same automorphism group as $x$.

Consider the fiber square.

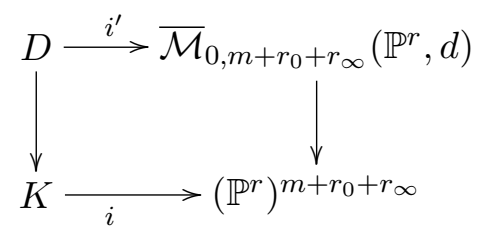

Now, the refined Gysin map $i^{!}: A_{j}\left(\overline{\mathcal{M}}_{0, m+r_{0}+r_{\infty}}\left(\mathbb{P}^{r}, d\right)\right) \longrightarrow A_{j-l}(D)$ satisfies

$$
\operatorname{deg}\left(i^{!}\left[\overline{\mathcal{M}}_{0, m+r_{0}+r_{\infty}}\left(\mathbb{P}^{r}, d\right)\right]^{\operatorname{vir}}\right)=\operatorname{deg}\left(\operatorname{Ev}^{*}(c) \cap\left[\overline{\mathcal{M}}_{0, m+r_{0}+r_{\infty}}\left(\mathbb{P}^{r}, d\right)\right]^{\text {vir }}\right) .
$$

But since $K$ is a regularly embedded substack, $i^{!}=\left(i^{\prime}\right)^{*}$.

Now, we need the following fact that ensures the compatibility of the Gysin map with the virtual cycle construction [17, 3.9].

Lemma 5.8. Let $\xi: X_{0} \rightarrow X$ be a regular embedding of codimension $d, W$ a scheme, and $W_{0}$ a scheme defined by the following fiber square

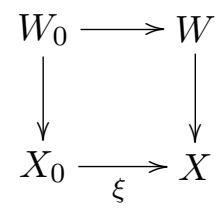

If the tangent obstruction complexes $\mathcal{T}_{W_{0}}^{\bullet}$ and $\mathcal{T}_{W}^{\bullet}$ are compatible in the sense of $([\mathbf{1 7}, 3.8])$ and certain technical assumptions are satisfied, then

$$
\xi^{!}[W]^{\mathrm{vir}}=\left[W_{0}\right]^{\mathrm{vir}} .
$$

It can be checked that the induced virtual cycle on $D$ is just $[D]^{\text {vir }}=[D]$. Now recall $([\mathbf{2 3}, 1.17])$, in the case of stacks over $\mathbb{C}$, for a map from an integral zero-dimensional stack $F$ to a point, $p$,

$$
\operatorname{deg}(F / p)=\frac{1}{\delta(F)}
$$

where $\delta(F)$ is the order of the automorphism group of $F$.

Therefore, the Gromov-Witten invariant is

$$
\operatorname{deg}\left(\left(i^{\prime}\right)^{*}\left[\overline{\mathcal{M}}_{0, m+r_{0}+r_{\infty}}\left(\mathbb{P}^{r}, d\right)\right]^{\mathrm{vir}}\right)=\operatorname{deg}(D / \mathrm{pt})=\sum_{F \in D} \frac{1}{\delta(F)} .
$$


Likewise, if $E$ is defined by

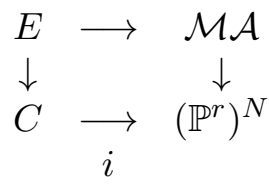

the rubber invariant is

$$
\operatorname{deg}\left(\left(i^{\prime}\right)^{*}[\mathcal{M} \mathcal{A}]^{\text {vir }}\right)=\operatorname{deg}(E / \mathrm{pt})=\sum_{F \in E} \frac{1}{\delta(F)} .
$$

The above theorem was proved in greater generality by a virtual localization argument in Andreas Gathmann's habilitation thesis [5, Theorem 5.2.7].

This result can be stated with greater succinctness following $([\mathbf{2}, 2.9 .2])$. Consider rubber invariants into $P=\mathbb{P}_{\mathbb{P}^{r}}(\mathcal{O}(n) \oplus \mathcal{O})$. The numerical condition for multiplicities implies

$$
\left(s_{1}^{0}+\cdots+s_{r_{0}}^{0}\right)-\left(s_{1}^{\infty}+\cdots+s_{r_{\infty}}^{\infty}\right)=n d .
$$

Choose a homogeneous basis $\left\{a_{1}, \ldots, a_{v}\right\}$ of $H^{*}(X)$. Choose variables $\beta_{i}$ dual to $a_{i}$ as in Definition 3.7. Let $p_{k, i}$ be the variables corresponding to contact to $D_{0}$ with multiplicity $k$, dual to $a_{i} \cdot q_{k, i}$ corresponds analogously to contact to $D_{\infty}$. Let $x$ be a real variable and define

$$
\begin{aligned}
P_{j} & =\sum_{k \geq 1} p_{k, j} e^{-i k x} \\
Q_{j} & =\sum_{k \geq 1} q_{k, j} e^{i k x} .
\end{aligned}
$$

Let $f$ be the rational Gromov-Witten potential of $\mathbb{P}^{r}$,

$$
\begin{aligned}
f\left(t_{1}, \ldots, t_{v}, z\right)= & \sum_{d} \sum_{n_{i}} \frac{1}{n_{1} ! \ldots n_{v} !}\left(\left(\operatorname{Ev}_{\overline{\mathcal{M}}}^{*}\left(\left(t_{1} a_{1}\right)^{n_{1}} \times \cdots \times\left(t_{v} a_{v}\right)^{n_{v}}\right)\right)\right. \\
& \left.\cap\left[\overline{\mathcal{M}}_{0, \sum n_{i}}\left(\mathbb{P}^{r}, d\right)\right]^{\text {vir }}\right) .
\end{aligned}
$$

Corollary 5.9. The rational rubber potential is given by

$$
A=\frac{1}{2 \pi} \int_{0}^{2 \pi} f\left(\beta_{1}+P_{1}+Q_{1}, \beta_{2}+P_{2}+Q_{2}, \ldots, \beta_{v}+P_{v}+Q_{v}, z e^{i m x}\right) d x
$$

where within the above formula, we treat $p_{k, i}, q_{k^{\prime}, i^{\prime}}$ as super-commuting variables of degree

$$
\begin{aligned}
\operatorname{deg} p_{k, i} & =2-2 \operatorname{deg} a_{i}-2 k \\
\operatorname{deg} q_{k^{\prime}, i^{\prime}} & =2-2 \operatorname{deg} a_{i^{\prime}}+2 k
\end{aligned}
$$

and the p-variables are to be written before the q-variables. 
Proof. The operation

$$
\frac{1}{2 \pi} \int_{0}^{2 \pi} d x
$$

has the effect neglecting all coefficients of $e^{m i x}$ for $m \neq 0$ which ensures that numerical condition for multiplicities is satisfied. It is clear then that the rubber invariant is equal to the corresponding Gromov-Witten invariant where interior and boundary marked points are treated as marked points.

This Fourier series formalism is similar to the residue formalism of [6].

5.2. Caporaso-Harris formula. Here we examine rubber invariants of $\mathcal{O}(1)$ over $\mathbb{P}^{1}$ without powers of $\mathrm{L}^{0}$ or $\mathrm{L}^{\infty}$.

Lemma 5.10. All higher genus $(g \geq 1)$ rubber invariants of $L=\mathcal{O}(1)$ over $X=\mathbb{P}^{1}$ with at least one point of tangency to $D_{\infty}$ and one interior marked point vanish.

Proof. This is a virtual dimension count.

Now, following [2], let us apply Corollary 5.9 to compute the rational rubber potential. Let us change notation slightly and write a basis for $H^{*}\left(\mathbb{P}^{1}\right)$ as

$$
\begin{aligned}
& a_{0} \in H^{0}\left(\mathbb{P}^{1}\right) \\
& a_{2} \in H^{2}\left(\mathbb{P}^{1}\right)
\end{aligned}
$$

and write $t_{0}, t_{2}$ for variables dual to $a_{0}, a_{2}$. The rational Gromov-Witten potential for $\mathbb{P}^{1}$ is

$$
f_{\mathbb{P}^{1}}\left(t_{0}, t_{2}, z\right)=\frac{t_{0}^{2} t_{2}}{2}+e^{t_{2}} z .
$$

Therefore, the rubber potential, $A$ satisfies

$$
\begin{aligned}
A_{2} \equiv & \left.\frac{\partial A}{\partial \beta_{2}}\right|_{\beta_{2}=0}=\hbar^{-1}\left(\beta_{0}^{2}+\sum_{k} p_{k, 0} q_{k, 0}\right. \\
& \left.+\frac{1}{2 \pi} \int_{0}^{2 \pi} e^{\sum_{k} p_{k, 2} e^{-i k x}+\sum_{k} q_{k, 2} e^{i k x}+i x} z d x\right) .
\end{aligned}
$$

Now we write down the relative potential of $\left(\mathbb{P}^{2}, L\right)$, the projective plane relative a line. Let $\left\{H^{2}, H^{1}, 1\right\}$ be a basis of $H^{*}\left(\mathbb{P}^{2}\right)$. We restrict ourselves to the potential involving only cohomology classes of the form $H^{2}$ and at least one $p$ variable. Use $\theta_{1}$ to express the element of $\mathcal{F}$ dual to $H^{2}$ and use $p_{n, 0}$ and $p_{n, 2}$ to express $n$th order multiplicities to $H$ at $[L]$ and $[\mathrm{pt}] \in H^{*}(L)$, respectively. Write the degree as $z^{d}$, where $d$ denotes the class $d[L] \in H_{2}\left(\mathbb{P}^{2}\right)$. Therefore, $F$ is an expression in $\tilde{\hbar}, \theta_{1}, \tilde{p}_{n, 0}, \tilde{p}_{n, 2}$, and $z^{d}$. 
By dimensional considerations, $\left.F\right|_{\theta_{1}=0}=0$ and the differential equation of Theorem 4.15 becomes

$$
\frac{\partial F}{\partial \theta_{1}}=A_{2} \cdot F
$$

Unwinding the action of $\mathcal{R}$ on $\mathcal{F}$, we see that this becomes

$$
\begin{aligned}
\frac{\partial F}{\partial \theta_{1}}= & \left(\sum_{k} k \tilde{p}_{k, 0} \frac{\partial}{\partial \tilde{p}_{k, 2}}\right. \\
& \left.+\hbar^{-1} \frac{1}{2 \pi} \int_{0}^{2 \pi} e^{\sum_{k} \tilde{p}_{k, 2} e^{-i k x}+\sum_{k} k \tilde{\hbar} \partial / \partial \tilde{p}_{k, 0} e^{i k x}+i x} z d x\right) F .
\end{aligned}
$$

This is the Caporaso-Harris formula as written in [6].

5.3. Ruled surfaces $\mathbb{F}_{n}$. We can apply the rubber formalism to derive the inductive formula for the relative Gromov-Witten invariants on Hirzebruch surfaces from $[\mathbf{2 1}]$. Let $\mathbb{F}_{n}$ be the ruled surface

$$
\mathbb{F}_{n}=\mathbb{P}_{\mathbb{P}^{1}}(\mathcal{O}(n) \oplus \mathcal{O}),
$$

where $n \geq 0$. Let $\pi$ be the projection $\pi: \mathbb{F}_{n} \rightarrow \mathbb{P}^{1}$.

Let $D \subset \mathbb{F}_{n}$ be the infinity section of $\mathcal{O}_{\mathbb{P}^{1}}(n)$. The second homology of $\mathbb{F}_{n}, H_{2}\left(\mathbb{F}_{n}\right)$ is generated by $C_{0}=D$, and $f$, a fiber of $\pi$.

Again, let us consider the terms in $F$ with at least one $p$-variable and no point classes at interior marked points. By dimensional reasons, the only non-vanishing invariant comes from degree 1 maps to a fiber. In this case, the virtual cycle of the moduli space agrees with the usual fundamental cycle, yielding

$$
\left.F\right|_{\theta_{1}=0}=\tilde{p}_{1,2} z^{f} .
$$

Now, we can compute the rubber invariants of $X=\mathbb{P}^{1}, L=N_{D / \mathbb{F}_{n}}=$ $\mathcal{O}(-n)$. By the same arguments as above,

$$
A_{2}=\hbar^{-1}\left(\beta_{0}^{2}+\sum_{k} p_{k, 0} q_{k, 0}+\frac{1}{2 \pi} \int_{0}^{2 \pi} e^{\sum_{k} p_{k, 2} e^{-i k x}+\sum_{k} q_{k, 2} e^{i k x}-i n x} z d x\right) .
$$

We get the differential equation

$$
\frac{\partial F}{\partial \theta_{1}}=\left(\sum_{k} k \tilde{p}_{k, 0} \frac{\partial}{\partial \tilde{p}_{k, 2}}+\hbar^{-1} \frac{1}{2 \pi} \int_{0}^{2 \pi} e^{\sum_{k} \tilde{p}_{k, 2} e^{-i k x}+\sum_{k} k \tilde{\hbar} \frac{\partial}{\partial \tilde{p}_{k, 0}} e^{i k x}-i n x} z^{C_{0}} d x\right) F .
$$

Under the identification

$$
\mathrm{Bl}_{0} \mathbb{P}^{2}=\mathbb{F}_{1}
$$

the above recursion formula reduces to Ran's $[\mathbf{2 0}]$. 
5.4. Rational Gromov-Witten invariants for $\mathbb{P}^{n}$. Here we consider the rational relative Gromov-Witten invariants of $\left(\mathbb{P}^{n}, H\right)$, where $H$ is a hyperplane in $\mathbb{P}^{n}$ and $n \geq 3$. We follow the computation of $[\mathbf{2}]$ which gives the formula of [22]. Let us compute the potential $F$ where we count positive degree curves and at interior marked points we consider only cohomology classes from $H^{k}\left(\mathbb{P}^{n}\right)$ for $k \geq 4$. Let $e_{4}, e_{6}, \ldots, e_{2 n} \in H^{*}\left(\mathbb{P}^{n}\right)$ be defined by $e_{2 i}=[H]^{i}$. Let $\theta_{2 i}$ be dual to $e_{2 i}$. On $\mathbb{P}^{n-1}$, we have cohomology classes $c_{0}, c_{2}, \ldots, c_{2 n-2}$, where $c_{2 i}$ generates $H^{2 i}\left(\mathbb{P}^{n-1}\right)$. Let $\tilde{p}_{k, i}$ be dual to $c_{2 i}$.

By dimensional considerations,

$$
\left.F\right|_{\theta_{4}=\cdots=\theta_{2 n}=0}=0 .
$$

Let

$$
f_{\mathbb{P}^{n-1}}\left(t_{0}, t_{2}, \ldots, t_{2 n-2}, z\right)
$$

be the rational Gromov-Witten potential of $\mathbb{P}^{n-1}$ where $t_{2 i}$ is dual to a cohomology class in $H^{2 i}\left(\mathbb{P}^{n-1}\right)$ and $z$ is the degree marker. Then, we can use Corollary 5.9 to write the rubber potential,

$A_{2 i}=\frac{1}{2 \pi} \int_{0}^{2 \pi} \frac{\partial f}{\partial t_{2 i}}\left(\beta_{1}+P_{1}+Q_{1}, \beta_{2}+P_{2}+Q_{2}, \ldots, \beta_{v}+P_{v}+Q_{v}, z e^{i x}\right) d x$.

Our differential equations become

$$
\frac{\partial F}{\partial \theta_{2 i}}=A_{2 i-2} \cdot F
$$

\section{Hamiltonian formalism}

In this section, we study a formalism for relative Gromov-Witten invariants analogous to the SFT [2] of Eliashberg, Givental, and Hofer. This formalism has the advantage that it takes into account some of the redundancies of rubber invariants given by Theorem 4.16. The rubber invariants are encoded in a certain generating function called the Hamiltonian.

Let $X$ be a projective manifold and let $P=\mathbb{P}_{X}\left(L \oplus 1_{X}\right)$ be the projective completion of a line-bundle $L$ over $X$. Let $\mathcal{M A}$ be a rubber moduli stack of maps to $P$ relative the zero and infinity sections with evaluation map

$$
\mathrm{Ev}: \mathcal{M A} \longrightarrow X^{m} \times X^{r_{0}} \times X^{r_{\infty}}
$$

where the three factors in the target denote the image of $m$ interior marked points, $r_{0}$ boundary marked points evaluating to the zero section and $r_{\infty}$ boundary marked points evaluating to the infinity section.

In SFT, there is a similar moduli space, $\mathcal{M}$. The construction and compactification of this moduli space are markedly different and the evaluation map is

$$
\mathrm{Ev}: \mathcal{M} \longrightarrow(S(L))^{m} \times X^{r_{0}} \times X^{r_{\infty}}
$$


where $S(L)$ is the unit circle bundle in $L$. Consequently, the classes that are pulled back at interior marked points are from $H^{*}(S(L))$. The cohomology of $S(L)$ is related to that of $X$ by the following Gysin sequence:

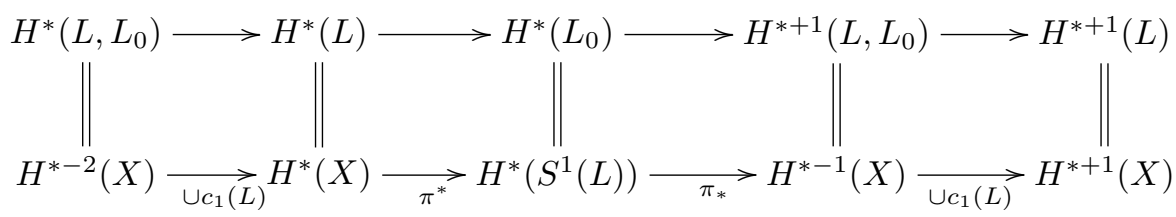

where $\pi: S(L) \rightarrow X$.

Classes in $H^{*}\left(S^{1}(L)\right)$ are non-canonically decomposable into classes of two kinds; those in the image of $\pi^{*}$; those in the cokernel of $\pi^{*}$. Classes in the image of $\pi^{*}$ do not fix the $S^{1}$-symmetry of the fibers in $S^{1}(L)$ while classes in the cokernel of $\pi^{*}$ do. Classes in the cokernel, we call phasefixing while those in the image we call non-phase-fixing. There is a subset of SFT invariants that involve pulling back phase-fixing classes at exactly one marked point. We replicate this theory using relative Gromov-Witten invariants. We believe that the analog of full SFT in which we use a number of phase-fixing classes is the theory as below but enriched by powers of $c_{1}\left(\mathrm{~L}^{\infty}\right)$.

Consider the following two types of marked point depending on the type of cohomology classes that is pulled back along their evaluation maps:

(1) Phase-fixing marked points $\phi$ that involve a class of the form $\operatorname{ev}_{\phi}^{*}\left(\pi_{*} a\right)$ or, equivalently, a class $\operatorname{ev}_{\phi}^{*} b$ where

$$
b \in \operatorname{ker}\left(\cup c_{1}(L): H^{*}(X) \longrightarrow H^{*+2}(X)\right) ;
$$

(2) Non-phase fixing marked points $i$ that involve $\operatorname{ev}_{i}^{*}(a)$, where $a$ is considered as a representative of an element in $\operatorname{coker}\left(\cup c_{1}(L): H^{*-2}(X) \rightarrow\right.$ $\left.H^{*}(X)\right)$.

6.1. Definition of Hamiltonian. We describe a particular kind of basis for the cohomology of $X$. The Hamiltonian will be dependent on the choice of basis in a predictable way.

Definition 6.1. A phase fixing basis is a homogeneous basis $\left\{a_{1}, \ldots, a_{m}\right\}$ for $\operatorname{ker}\left(\cup c_{1}(L): H^{*}(X) \rightarrow H^{*+2}(X)\right)$.

Definition 6.2. A non-phase fixing basis is a set of homogeneous elements $\left\{b_{1}, \ldots, b_{n}\right\}$ of $H^{*}(X)$ that descend to a basis of $\operatorname{coker}\left(\cup c_{1}(L): H^{*-2}(X) \rightarrow\right.$ $\left.H^{*}(X)\right)$.

We define $\mathcal{H}$, a graded super-Weyl algebra over $\mathbb{Q}\left[H_{2}(X)\right]$. Choose a phase-fixing basis $\left\{a_{1}, \ldots, a_{m}\right\}$, a non-phase fixing basis $\left\{b_{1}, \ldots, b_{n}\right\}$, and a homogeneous basis $\left\{c_{1}, \ldots, c_{s}\right\}$ of $H^{*}(X)$. We will consider formal variables $\sigma_{i}, \tau_{j}$ dual to $a_{i}, b_{j}$, respectively. For every positive integer $n$, we will have 
$p_{n, i}, q_{n, i}$ dual to $c_{i}$. Let $\mathcal{H}$ be a graded algebra generated by the following elements

$$
\begin{aligned}
\operatorname{deg} \hbar & =-2(\operatorname{dim} X-2) \\
\operatorname{deg} z^{d} & =2\left\langle c_{1}(T X), d\right\rangle+2\left\langle c_{1}(L), d\right\rangle \\
\operatorname{deg} \sigma_{i} & =-1-\operatorname{deg} a_{i} \\
\operatorname{deg} \tau_{i} & =2-\operatorname{deg} b_{i} \\
\operatorname{deg} p_{n, i} & =2-\operatorname{deg} c_{i}-2 n \\
\operatorname{deg} q_{n, i} & =2-\operatorname{deg} c_{i}+2 n .
\end{aligned}
$$

In $\mathcal{H}, \hbar, z^{A}, \sigma_{i}, \tau_{i}$ are super-central while the $p$ - and $q$-variables satisfy the following super-commutation relations:

$$
\left[p_{n_{1}, i_{1}}, p_{n_{2}, i_{2}}\right]=0, \quad\left[q_{n_{1}, i_{1}}, q_{n_{2}, i_{2}}\right]=0, \quad\left[q_{n_{1}, i_{1}}, p_{n_{2}, i_{2}}\right]=n_{1} \delta_{n_{1}, n_{2}} g^{i_{1} i_{2}} \hbar .
$$

As in $\mathcal{R}$, the algebra $\mathcal{H}$ consists of Laurent series in $\hbar$ whose coefficients are power series in the $p$-variables whose coefficients are power series in the $\tau$ - variables whose coefficients are polynomials in the $\sigma$ - and $q$-variables.

Note that we have changed the grading of $\sigma_{i}$ from what we would have expected in the definition of the rubber potential.

To define the Hamiltonian, define the following formal sums:

$$
\begin{aligned}
S & =\sum_{n} \sigma_{i} a_{i} \\
T & =\sum_{n} \frac{1}{n !}\left(\sum_{i} \tau_{i} b_{i}\right)^{n} \\
P & =\sum_{n} \frac{1}{n !}\left(\sum_{k, i} p_{k, i}\right)^{n} \\
Q & =\sum_{n} \frac{1}{n !}\left(\sum_{k, i} q_{k, i}\right)^{n} .
\end{aligned}
$$

Definition 6.3. The Hamiltonian $H$ is

$$
H=\sum_{\chi} \sum_{d \in H_{2}(X)} \hbar^{-\frac{1}{2} \chi} z^{d}(S \cdot T, P, Q)_{\chi, d, 0},
$$

where $S \cdot T$ denotes formal multiplication.

Below we may suppress $P$ and $Q$ in the correlator.

$H$ is linear in the $\sigma$-variables which keep track of the phase-fixing classes which are evaluated at a single, distinguished marked point. $H$ is homogeneous of degree -1 . 
6.2. Dependence on representatives. We will show how the Hamiltonian depends on the choice of representative classes in coker $\left(\cup c_{1}(L): H^{*-2}(X) \rightarrow\right.$ $\left.H^{*}(X)\right)$.

Lemma 6.4. Consider $\mathcal{M A}$ where we distinguish two interior marked points, $\phi$ and $i$. If $a \in \operatorname{ker}\left(\cup c_{1}(L): H^{*}(X) \rightarrow H^{*+2}(X)\right)$ then

$$
\mathrm{ev}_{\phi}^{*}(a) \mathrm{ev}_{i}^{*}\left(c_{1}(L)\right)=\mathrm{ev}_{\phi}^{*}(a)\left(f_{-i}-\phi f_{\phi}\right) .
$$

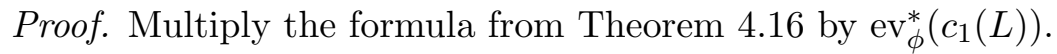

If we view $\phi$ as the phase-fixing marked point, every term in the Hamiltonian will involve a factor of the form $\operatorname{ev}_{\phi}^{*} a$, where $a \cup c_{1}(L)=0$.

Choose a phase-fixing basis,

$$
\left\{a_{1}, \ldots, a_{m}\right\} \subset H^{*}(X)
$$

and a non-phase fixing basis

$$
\left\{b_{1}, \ldots, b_{n}\right\} \subset H^{*}(X) .
$$

From the construction of $\mathcal{H}$, the Hamiltonian is invariant under change of basis of the form

$$
b_{i}^{\prime}=M_{i j} b_{j}
$$

Therefore, we need only determine how $H$ varies when we change $b_{i} \in H^{*}(X)$ within its equivalence class $\left[b_{i}\right] \in \operatorname{coker}\left(\cup c_{1}\right)$. Let us change $\left\{b_{1}, \ldots, b_{n}\right\}$ one element at a time. Write

$$
b_{1}^{\prime}-b_{1}=d_{1} c_{1}(L) .
$$

Let $\tilde{b}_{t}=(1-t) b_{1}+t b_{1}^{\prime}$ and $H_{t}$ be $H$ with $\tilde{b}_{t}$ substituted for $b_{1}$, so $H_{0}$ is the Hamiltonian with $b_{1}$ in place, $H_{1}$ is the Hamiltonian with $b_{1}^{\prime}$ in place.

$$
\begin{aligned}
H_{t}= & \sum_{\Gamma} \frac{1}{\operatorname{Aut}_{\Gamma}\left(R_{0}\right) \operatorname{Aut}_{\Gamma}\left(R_{\infty}\right) k_{1} ! \ldots k_{n} !}\left(a_{j} \cdot \tilde{b}_{t}^{k_{1}} \cdot b_{2}^{k_{2}} \ldots b_{n}^{k_{n}}\right)_{\chi, A, 0} \\
& \cdot \hbar^{\frac{\chi}{2}} \sigma_{i} \tau_{1}^{k_{1}} \ldots \tau_{n}^{k_{n}} p^{\Gamma} q^{\Delta} z^{A}
\end{aligned}
$$

SO

$$
\begin{aligned}
\frac{\partial H_{t}}{\partial t}= & \sum_{\Gamma} \frac{1}{\operatorname{Aut}_{\Gamma}\left(R_{0}\right) \operatorname{Aut}_{\Gamma}\left(R_{\infty}\right) k_{1} ! \ldots k_{n} !} \\
& \cdot\left(a_{j} \cdot k_{1}\left(d_{1} c_{1}(L)\right) \cdot \tilde{b}_{t}^{k_{1}-1} \cdot b_{2}^{k_{2}} \ldots b_{n}^{k_{n}}\right)_{\chi, d, 0} \hbar^{-\frac{\chi}{2}} \sigma_{i} \tau_{1}^{k_{1}} \ldots \tau_{n}^{k_{n}} p^{\Gamma} q^{\Delta} z^{A} .
\end{aligned}
$$


But by the lemma

$$
\begin{aligned}
& \left(a_{j} \cdot\left(d_{1} c_{1}(L)\right) \cdot \tilde{b}_{t}^{k_{1}-1} \cdot b_{2}^{k_{2}} \ldots b_{n}^{k_{n}}\right)_{\chi, d, 0} \\
& \quad \operatorname{Ev}^{*}\left(a_{j} \times d_{1} \times \tilde{b}_{t}^{k_{1}-1} \times b_{2}^{k_{2}} \times \cdots \times b_{n}^{k_{n}}\right) \cdot\left(\begin{array}{cc}
f & \phi \\
- & \\
-\phi & 1
\end{array}\right)
\end{aligned}
$$

where the first two entries in Ev corresponds to the marked points denoted by $\phi$ and 1 , respectively.

Let us define the generating function $K_{t}$ by

$$
\begin{aligned}
K_{t}= & \sum_{\Gamma} \frac{1}{\operatorname{Aut}_{\Gamma}\left(R_{0}\right) \operatorname{Aut}_{\Gamma}\left(R_{\infty}\right) k_{1} ! \ldots k_{n} !} \\
& \cdot\left(d_{1} \cdot \tilde{b}_{t}^{k_{1}} \cdot b_{2}^{k_{2}} \ldots b_{n}^{k_{n}}\right)_{\chi, d, 0} \hbar^{\chi / 2} \tau_{1}^{k_{1}+1} \ldots \tau_{n}^{k_{n}} p^{\Gamma} q^{\Delta} z^{d} .
\end{aligned}
$$

Because $K$ is of even degree,

$$
\frac{\partial H_{t}}{\partial t}=\left[K_{t}, H_{t}\right]
$$

6.3. The Hamiltonian as a differential. We may define a differential, $D^{H}$, on the algebra $\mathcal{H}$. The homology with respect to $D^{H}$ will be an invariant of $(X, L)$.

Lemma 6.5. Consider $\mathcal{M A}$ with at least two marked points including $\phi_{1}$ and $\phi_{2}$. Let $a_{1}, a_{2} \in \operatorname{ker}\left(\cup c_{1}(L): H^{*}(X) \rightarrow H^{*+2}(X)\right)$. Then

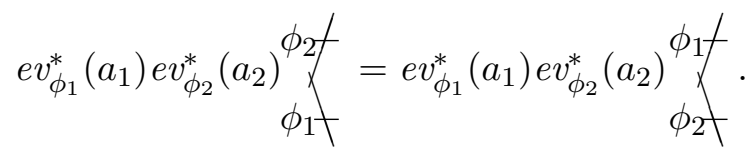

Proof. We intersect

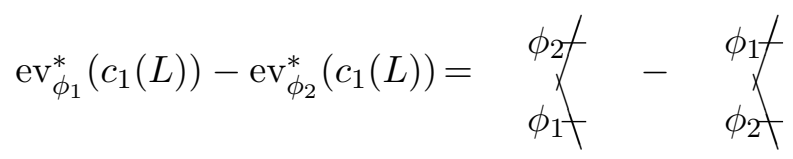

from Theorem 4.16 with $\operatorname{ev}_{\phi_{1}}^{*}\left(a_{1}\right) \mathrm{ev}_{\phi_{2}}^{*}\left(a_{2}\right)$.

Theorem 6.6. $H$ is nilpotent of order 2 .

Proof. Write

$$
H=\sum_{i} \sigma_{i} H^{i}
$$


Notice that $H^{i}$ is an element of degree $1-\operatorname{deg} \sigma_{i}=2+\operatorname{deg} a_{i}$.

$$
\begin{aligned}
H^{2}= & \left(\sum_{i} \sigma_{i} H^{i}\right)^{2} \\
= & \sum_{i} \sigma_{i} H^{i} \sigma_{i} H^{i}+\sum_{i<j}\left(\sigma_{i} H^{i} \sigma_{j} H^{j}+\sigma_{j} H^{j} \sigma_{i} H^{i}\right) \\
= & \sum_{i} \sigma_{i}^{2}\left(H^{i}\right)^{2}+\sum_{i<j}\left(\sigma _ { i } \sigma _ { j } \left((-1)^{\left(\operatorname{deg} a_{i}\right)\left(\operatorname{deg} a_{j}-1\right)} H^{i} H^{j}\right.\right. \\
& \left.\left.+(-1)^{\left(\operatorname{deg} a_{i}-1\right)\left(\operatorname{deg} a_{j}\right)+\left(\operatorname{deg} a_{i}-1\right)\left(\operatorname{deg} a_{j}-1\right)} H_{j} H_{i}\right)\right) .
\end{aligned}
$$

The only way it can be that $\sigma_{i}^{2} \neq 0$ is if $\operatorname{deg} \sigma_{i}$ is even, and therefore, $\operatorname{deg} a_{i}$ is odd. In this case, we must show $\left(H^{i}\right)^{2}=0$. By arguments analogous to the proof of the degeneration formulae, $\left(H^{i}\right)^{2}$ is given by

$$
H_{i}^{2}=\left(\operatorname{ev}_{\phi_{1}}^{*}\left(a_{1}\right) \operatorname{ev}_{\phi_{2}}^{*}\left(a_{2}\right)(\text { sum over terms }) \cup \underset{\phi_{1}}{\phi_{2}} \underset{f}{f}\right) \cap[\mathcal{M} \mathcal{A}]^{\mathrm{vir}} .
$$

where "sum over terms" indicate a sum over all the cohomology classes and formal variables that enter into Definition 6.3. By the above lemma, this equals

$$
\begin{aligned}
& \left(\operatorname{ev}_{\phi_{1}}^{*}\left(a_{i}\right) \operatorname{ev}_{\phi_{2}}^{*}\left(a_{i}\right)(\text { sum over terms }) \cup \underset{\phi_{2}}{\phi_{1}} \underset{f}{ }\right) \cap[\mathcal{M} \mathcal{A}]^{\mathrm{vir}} \\
& =-\left(\operatorname{ev}_{\phi_{2}}^{*}\left(a_{i}\right) \operatorname{ev}_{\phi_{1}}^{*}\left(a_{i}\right)(\text { sum over terms }) \cup \underset{\phi_{1}}{\phi_{2}} \gamma^{\prime}\right) \cap[\mathcal{M} \mathcal{A}]^{\mathrm{vir}}=-H_{i}^{2} .
\end{aligned}
$$

Since for $i \neq j, \sigma_{i} \sigma_{j} \neq 0$, we must show

$$
\begin{aligned}
0 & =(-1)^{\left(\operatorname{deg} a_{i}\right)\left(\operatorname{deg} a_{j}-1\right)} H^{i} H^{j}+(-1)^{\left(\operatorname{deg} a_{i}-1\right)\left(\operatorname{deg} a_{j}\right)+\left(\operatorname{deg} a_{i}-1\right)\left(\operatorname{deg} a_{j}-1\right)} H^{j} H^{i} \\
& =(-1)^{\operatorname{deg} a_{i}-1}\left(H^{j} H^{i}-(-1)^{\left(\operatorname{deg} a_{i}\right)\left(\operatorname{deg} a_{j}\right)} H^{i} H^{j}\right) .
\end{aligned}
$$

The proof is similar to the one above.

Definition 6.7. The operator $D^{H}: \mathcal{H} \rightarrow \mathcal{H}$ is defined on homogeneous elements of $\mathcal{H}$ by $D^{H}: f \mapsto H f-(-1)^{\operatorname{deg} f} f H$ and extended by linearity.

Corollary 6.8. $D^{H}$ is a differential on the graded algebra $\mathcal{H}$

Proof. It is straightforward to show $\left(D^{H}\right)^{2}=0$ and

$$
D^{H}(f g)=D^{H}(f) g+(-1)^{\operatorname{deg} f} f D^{H}(g) .
$$


6.4. Hamiltonian homology. We show that the chain complex $\left(\mathcal{H}, D^{H}\right)$ is invariant under changing the choice of representatives of coker $\left(\cup c_{1}(L)\right)$ in the non-phase fixing basis.

Theorem 6.9. Let $\tilde{b}_{t}=(1-t) b_{1}+t b_{1}^{\prime}$. Let $H_{t}$ be $H$ with $\tilde{b}_{t}$ substituted for $b_{1}$. Then there is a family of isomorphisms $Q_{t}: \mathcal{H} \rightarrow \mathcal{H}$ so that

$$
D^{H_{t}} Q_{t}(f)=Q_{t}\left(D^{H} f\right) .
$$

Proof. Let $K_{t}$ be as above. Define $A_{t}$ by

$$
A_{t}=\int_{0}^{t} K_{s} d s
$$

and let $Q_{t}$ be given by

$$
Q_{t}(f)=e^{A_{t}} f e^{-A_{t}}
$$

Write $f_{t}=Q_{t}(f)$ and let

$$
E_{t}=D^{H_{t}}\left(f_{t}\right)-Q_{t}\left(D^{H_{0}} f\right) .
$$

Therefore,

$$
\begin{aligned}
E_{t} & =\left[H_{t}, f_{t}\right]-Q_{t}\left(\left[H_{0}, f\right]\right), \\
\frac{\partial E_{t}}{\partial t} & =\left[\left[K_{t}, H_{t}\right], f_{t}\right]+\left[H_{t},\left[K_{t}, f_{t}\right]\right]-\left[K_{t}, Q_{t}([H, f])\right] \\
& =\left[K_{t}, E_{t}\right] .
\end{aligned}
$$

Since $E_{0}=0$, it follows that $E_{t}=0$.

By changing representatives of coker $\left(\cup c_{1}(L)\right)$ one-by-one, we see that the differential graded algebra and hence the Hamiltonian homology is invariant.

There are other versions of the Hamiltonian homology, rational Hamiltonian homology and contact Hamiltonian homology. They bear the same relation to Hamiltonian homology as their analogs do to SFT homology. We refer the reader to $[\mathbf{2}]$ for details.

\section{Localization proof of degeneration formula}

In this section, we give a proof of the degeneration formula in Theorem 4.14. That degeneration formula is the generating function interpretation of the following relation among line-bundles on $\mathcal{M A}$ :

$$
\mathrm{L}^{\infty} \otimes \mathrm{ev}_{i}^{*} L=\mathrm{L}_{i, \text { not bot }} .
$$

A proof is outlined above, but here we give a more direct proof using the virtual localization technique from $[\mathbf{1 2}]$ and $[\mathbf{8}]$ and adapted for the relative case in $[\mathbf{9}]$.

Our strategy is to evaluate the equivariant cap product,

$$
\left.\hbar \cup \operatorname{Ev}^{*} c \cup \operatorname{ev}_{i}^{*}\left(c_{1}(\mathcal{O}(1))+c_{1}(L)\right)\right) \cap[\mathcal{M Y}]^{\mathrm{vir}} .
$$


which we know to be zero on a particular stack, $\mathcal{M Y}$ by localization. The localization formula a relation among cycle classes which when intersected with cohomology classes yields Theorem 4.14.

7.1. Target schemes. We need to construct a stack $\mathcal{M Y}$ that is closely related to $\mathcal{M A}$. Let $X$ be a projective manifold and $L$ be a line-bundle over $X$. Let $P=\mathbb{P}_{X}\left(L \oplus 1_{X}\right)$. Let $p: P \rightarrow X$ be the projection. Let $i_{0}: D_{0} \rightarrow$ $P$ and $i_{\infty}: D_{\infty} \rightarrow P$ be the inclusions of the zero and infinity sections, respectively.

We want to consider stable maps into $P$ relative $D_{0}$ and $D_{\infty}$. The stack of stable maps we construct will differ from $\mathcal{M A}$, in that we do not have a $\mathbb{C}^{*}$-action that dilates the fiber of $P$.

Define the scheme ${ }_{k, l} Y$ as the union of $k+l+1$ copies of $P$,

$$
{ }_{k, l} Y=P_{-k} \sqcup_{X} \ldots \sqcup_{X} P_{-1} \sqcup_{X} P_{0} \sqcup_{X} P_{1} \sqcup_{X} \ldots \sqcup_{X} P_{l}
$$

where $X_{0} \subset P_{i}$ is identified with $X_{\infty} \subset P_{i+1}$. Let the automorphism group of ${ }_{k, l} Y$ be $\left(\mathbb{C}^{*}\right)^{k} \times\left(\mathbb{C}^{*}\right)^{l}$ where the first $k$ copies of $\mathbb{C}^{*}$ dilate the fibers of $P_{-k}, \ldots, P_{-1}$ and the last $l$ copies of $\mathbb{C}^{*}$ dilate the fibers of $P_{1}, \ldots, P_{l}$. Note that there is no $\mathbb{C}^{*}$-factor dilating $P_{0}$. $\mathcal{M Y}$ is the stack of stable predeformable maps to ${ }_{k, l} Y$ with data given by a rubber topological type.

The rigorous definition of $\mathcal{M Y}$ is analogous to those of $\mathcal{M A}$ and $\mathcal{M Z}$. Begin by defining triples $\left(Y[k, l], D_{0}[k, l], D_{\infty}[k, l]\right)$ indexed by a pair of nonnegative integers where $Y[k, l]$ is a projective manifold with a $\left(\mathbb{C}^{*}\right)^{k} \times\left(\mathbb{C}^{*}\right)^{l}$ action, and $D_{0}[k, l]$ and $D_{\infty}[k, l]$ are smooth divisors. Let

$$
\begin{aligned}
Y[0,0] & =P \\
D_{0}[0,0] & =D_{0} \\
D_{\infty}[0,0] & =D_{\infty},
\end{aligned}
$$

where $D_{0}$ and $D_{\infty}$ are the zero and infinity sections in $P$. We define $Y[k, l]$ inductively,

$$
Y[k+1, l]=\mathrm{Bl}_{D_{\infty}[k, l] \times\{0\}}\left(Y[k, l] \times \mathbb{A}^{1}\right) .
$$

$D_{\infty}[k+1, l]$ is the proper transform of $D_{\infty}[k, l] \times \mathbb{A}^{1}, D_{0}[k+1, l]$ is the inverse image of $D_{0}[k, l] \times \mathbb{A}^{1}$.

$$
Y[k, l+1]=\mathrm{Bl}_{D_{0}[k, l] \times\{0\}}\left(Y[k, l] \times \mathbb{A}^{1}\right) .
$$

$D_{0}[k+1, l]$ is the proper transform of $D_{0}[k, l] \times \mathbb{A}^{1}, D_{\infty}[k+1, l]$ is the inverse image of $D_{\infty}[k+1, l] \times \mathbb{A}^{1}$. The $\left(\mathbb{C}^{*}\right)^{k} \times\left(\mathbb{C}^{*}\right)^{l}$ actions dilates the fibers in the tails.

Given a rubber topological type $\Gamma$, we construct $\mathcal{M Y}=\mathcal{M}(\mathcal{Y}, \Gamma)$ by mimicking the construction of $\mathcal{M Z}$ and $\mathcal{M A}$. We consider families of predeformable relative maps described by $\Gamma$ that are stable under the $\left(\mathbb{C}^{*}\right)^{k} \times$ $\left(\mathbb{C}^{*}\right)^{l}$-action. We glue these families together into a stack and then quotient by the $\left(\mathbb{C}^{*}\right)^{k} \times\left(\mathbb{C}^{*}\right)^{l}$-action. $\mathcal{M Y}$ carries a virtual cycle. 
Note that $\mathcal{M Y}$ is different from $\mathcal{M Z}\left(P, D_{0} \sqcup D_{\infty}\right)$ since in the construction of $Z[n], D_{0}$ and $D_{\infty}$ are blown up simultaneously and the $\mathbb{C}^{*}$-action dilates the fibers of their exceptional divisors together.

7.2. Equivariant data. We will perform a virtual localization computation on $\mathcal{M Y}$. We first define a $\mathbb{C}^{*}$ action on $P$. Specify points of $P=\mathbb{P}_{X}(L \oplus 1)$ by $[l: t]$. Define the group action by, for $\lambda \in \mathbb{C}^{*}$,

$$
\lambda \cdot[l: t]=[\lambda l: t] .
$$

This $\mathbb{C}^{*}$ action induces a $\mathbb{C}^{*}$-action on $\mathcal{M Y}$ so that

$$
\mathrm{Ev}: \mathcal{M Y} \longrightarrow P^{m} \times X^{r_{0}} \times X^{r_{\infty}}
$$

is equivariant.

Write $H_{\mathbb{C}^{*}}^{*}(\mathrm{pt})=\mathbb{C}[\hbar]$, where $\hbar$ is the Euler class of the equivariant line bundle on pt under the group action

$$
\lambda \cdot t=\lambda t
$$

Now, let $\Gamma$ be a rubber topological type where there is at least one vertex that does not correspond to a trivial cylinder. The proof of the virtual localization theorem holds for $\mathcal{M}(\mathcal{Y}, \Gamma)$ with straightforward modifications.

Let $\pi: P \rightarrow X$. Consider the composition $\left(\pi^{m} \times \operatorname{id}_{X^{r_{0}}} \times \operatorname{id}_{X^{r_{\infty}}}\right) \circ \mathrm{Ev}: \mathcal{M Y} \longrightarrow P^{m} \times X^{r_{0}} \times X^{r_{\infty}} \longrightarrow X^{m} \times X^{r_{0}} \times X^{r_{\infty}}$, and for $i$, an interior marked point, consider the evaluation map

$$
\mathrm{ev}_{i}: \mathcal{M Y} \longrightarrow P \text {. }
$$

Let $\mathcal{O}(1)$ be the equivariant line-bundle over $P$ that is dual to $\mathcal{O}(-1)$ equipped with the linearization

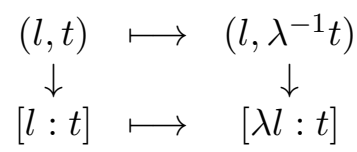

Note that $\mathcal{O}(1) \otimes p^{*} L$ is an equivariant line bundle whose first Chern class restricts to

$$
i_{0}^{*}\left(c_{1}(\mathcal{O}(1))+c_{1}\left(p^{*} L\right)\right)=\hbar+c_{1}(L) \in H_{\mathbb{C}^{*}}^{*}\left(D_{0}\right)=H^{*}\left(D_{0}\right)[\hbar]
$$

and

$$
i_{\infty}^{*}\left(c_{1}\left(\mathcal{O}(1)+c_{1}\left(p^{*} L\right)\right)=0 \in H_{\mathbb{C}^{*}}^{*}\left(D_{\infty}\right)=H^{*}\left(D_{\infty}\right)[\hbar] .\right.
$$

There is also a natural map pt* $: \mathbb{C}[\hbar]=H_{\mathbb{C}^{*}}^{*}(\mathrm{pt}) \rightarrow H_{\mathbb{C}^{*}}^{*}(\mathcal{M Y})$ and that $\mathrm{pt}^{*} \hbar$ (which we will denote by $\hbar$ ) is an equivariant extension of $0 \in H^{2}(\mathcal{M Y})$.

Let $n=\operatorname{vim} \mathcal{M Y}$ and $c \in H^{n-4}\left(X^{m} \times X^{r_{0}} \times X^{r_{\infty}}\right)$. Observe that

$$
\left.\operatorname{deg}\left(\left(\hbar \cup \operatorname{Ev}^{*} c \cup \operatorname{ev}_{i}^{*}\left(c_{1}(\mathcal{O}(1))+c_{1}\left(p^{*} L\right)\right)\right) \cap[\mathcal{M Y}]^{\operatorname{vir}}\right)\right|_{\hbar=0}=0
$$

because the cohomology class is an equivariant extension of 0 . We will prove Theorem 4.14 by computing this degree by virtual localization. 
7.3. Fixed loci. We identify the $\mathbb{C}^{*}$ fixed loci in $\mathcal{M Y}$. For a map $f$ in a $\mathbb{C}^{*}$ fixed locus, we have the composition

$$
C \longrightarrow{ }_{k, l} Y \longrightarrow P \text {. }
$$

The irreducible components of $C$ are of three types: (a) those mapping into $D_{0}$; (b) those mapping into $D_{\infty}$; (c) those mapping into a fiber of $P \rightarrow X$ totally ramified over two points. There are, therefore, three types of fixed loci:

(1) Those whose generic element only has components of type (a) and (c);

(2) Those whose generic elements only has components of type (b) and (c);

(3) Those whose generic element has components of all three types.

A fixed locus of the first type is parameterized by

$$
T: \mathcal{M}(\mathcal{A}, \Gamma) \longrightarrow F
$$

where the morphism $T$ attaches components of type (c) of degrees

$$
\left\{\mu^{\infty}(1), \ldots, \mu^{\infty}\left(\left|R_{\infty}\right|\right)\right\}
$$

to the boundary marked points. We have $T_{*}\left([\mathcal{M A}]^{\text {vir }}\right)=[F]^{\text {vir }}$. The evaluation maps fit into the commutative diagram

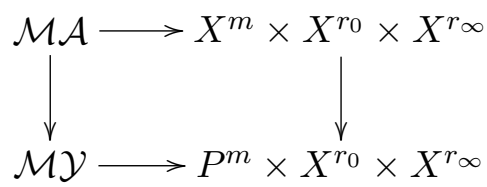

where the vertical map $X \rightarrow P$ is given by including $X$ as $D_{0} \subset P$. The virtual normal bundle to this fixed locus has Euler class

$$
e(N)=\hbar-c_{1}\left(\mathrm{~L}^{\infty}\right) .
$$

A fixed locus of the second type is parameterized by

$$
B: \mathcal{M}(\mathcal{A}, \Gamma) \longrightarrow F
$$

where the morphism $B$ attaches components of degrees

$$
\left\{\mu^{0}(1), \ldots, \mu^{0}\left(\left|R_{0}\right|\right)\right\} \text {. }
$$

The evaluation maps fit together as before except that the inclusion is now $D=D_{\infty} \hookrightarrow P . B_{*}\left([\mathcal{M A}]^{\text {vir }}\right)=[F]^{\text {vir }}$ and

$$
e(N)=-\hbar-c_{1}\left(\mathrm{~L}^{0}\right) .
$$

A fixed locus of the third type is parameterized by

$$
I: \mathcal{M}\left(\mathcal{A}, \Gamma_{A_{t}}\right) \times{ }_{D^{r}} \mathcal{M}\left(\mathcal{A}, \Gamma_{A_{b}}\right) \longrightarrow F
$$


corresponding to a type join quadruple $\left(\Gamma_{A_{t}}, \Gamma_{A_{b}}, L, J\right)$ such that $\Gamma_{A_{t}} *_{L, J}$ $\Gamma_{A_{b}}=\Gamma$. The morphism $I$ inserts components of type (c) of degrees

$$
\left\{\mu_{t}^{\infty}(1), \ldots, \mu_{t}^{\infty}\left(\mid R_{t \infty}\right)\right\}=\left\{\mu_{b}^{0}(1), \ldots, \mu_{b}^{0}\left(\left|R_{b 0}\right|\right)\right\}
$$

between the components coming from each factor of $\mathcal{M A}$. The evaluation map takes the interior marked points on $\mathcal{M}\left(\mathcal{A}, \Gamma_{A_{t}}\right)$ and $\mathcal{M}\left(\mathcal{A}, \Gamma_{A_{b}}\right)$ to $X=$ $D_{0} \subset P$ and $X=D_{\infty} \subset P$, respectively. We have

$$
I_{*}\left(\left[\mathcal{M}\left(\mathcal{A}, \Gamma_{A_{t}}\right) \times_{D^{r}} \mathcal{M}\left(\mathcal{A}, \Gamma_{A_{b}}\right)\right]^{\mathrm{vir}}\right)=\left|\operatorname{Aut}_{\Gamma_{A_{t}}, \Gamma_{A_{b}, L}}\left(R_{A_{t} \infty}, R_{A_{b} 0}\right)\right|[F]^{\mathrm{vir}} .
$$

If $p_{t}, p_{b}$ are the projections of $\mathcal{M}\left(\mathcal{A}, \Gamma_{A_{t}}\right) \times D_{D^{r}} \mathcal{M}\left(\mathcal{A}, \Gamma_{A_{b}}\right)$ onto each factor, then the normal bundle to the fixed locus has Euler class

$$
e(N)=\left(\hbar-p_{t}^{*} c_{1}\left(\mathrm{~L}^{\infty}\right)\right)\left(-\hbar-p_{b}^{*} c_{1}\left(\mathrm{~L}^{0}\right)\right) .
$$

7.4. Localization computation. We now compute the contribution from each fixed locus. The virtual localization formula [8] states that given a top-dimensional class $b \in H^{*}(\mathcal{M Y})$, we have

$$
\operatorname{deg}\left(b \cap[\mathcal{M Y}]^{\mathrm{vir}}\right)=\sum_{J: F \longrightarrow \mathcal{M Y}} \frac{1}{\operatorname{deg}(J)} \operatorname{deg}\left(\frac{J^{*} b}{e\left(N_{F}\right)} \cap[F]^{\mathrm{vir}}\right)
$$

where the sum is over fixed loci $F$ with parametrization $J: F \rightarrow \mathcal{M Y}$.

The fixed locus of the first type contributes

$$
\begin{gathered}
\operatorname{deg}\left(\frac{T^{*}\left(\hbar \mathrm{ev}_{i}^{*}\left(c_{1}(\mathcal{O}(1))+c_{1}(L)\right) \mathrm{Ev}^{*} c\right)}{e(N)} \cap[\mathcal{M} \mathcal{A}]^{\mathrm{vir}}\right) \\
=\operatorname{deg}\left(\frac{\hbar\left(\hbar+\mathrm{ev}_{i}^{*} c_{1}(L)\right)}{\hbar-c_{1}\left(\mathrm{~L}^{\infty}\right)} \mathrm{Ev}^{*} c \cap[\mathcal{M} \mathcal{A}]^{\mathrm{vir}}\right) \\
=\operatorname{deg}\left(\left(\mathrm{ev}_{i}^{*} c_{1}(L)+c_{1}\left(\mathrm{~L}^{\infty}\right)\right) \mathrm{Ev}^{*} c \cap[\mathcal{M} \mathcal{A}]^{\mathrm{vir}}\right) .
\end{gathered}
$$

Fixed loci of the second type do not contribute to the localization formula because $\mathrm{ev}_{i}: F \rightarrow P$ factors as

$$
\mathrm{ev}_{i}: F=\mathcal{M A} \longrightarrow X=D_{\infty} \hookrightarrow P
$$

and $i_{\infty}^{*}\left(c_{1}(\mathcal{O}(1))+c_{1}(L)\right)=0$.

The only fixed loci of the third type that contribute are those in which the $i$ th marked point is mapped to $D_{0}$. Such a fixed locus contributes

$$
\begin{aligned}
& \frac{1}{\mathrm{Aut}} \operatorname{deg}\left(\frac{I^{*}\left(\hbar \mathrm{ev}_{i}^{*}\left(c_{1}(\mathcal{O}(1))+c_{1}(L)\right) \mathrm{Ev}^{*} c\right)}{e(N)} \cap\left[\mathcal{M} \mathcal{A} \times{ }_{D^{r}} \mathcal{M} \mathcal{A}\right]^{\mathrm{vir}}\right) \\
& \quad=-\frac{1}{\mathrm{Aut}} \operatorname{deg}\left(\mathrm{Ev}^{*} c \cap\left[\mathcal{M} \mathcal{A} \times{ }_{D^{r}} \mathcal{M} \mathcal{A}\right]^{\mathrm{vir}}\right)
\end{aligned}
$$

where Aut $=\left|\operatorname{Aut}_{\Gamma_{A_{t}}, \Gamma_{A_{b}}, L}\left(R_{A_{t} \infty}, R_{A_{b} 0}\right)\right|$. 
Putting everything together we get

$$
\begin{aligned}
0= & \operatorname{deg}\left(\left(\mathrm{ev}_{i}^{*} c_{1}(L)+c_{1}\left(\mathrm{~L}^{\infty}\right)\right) \operatorname{Ev}^{*} c \cap[\mathcal{M} \mathcal{A}]^{\mathrm{vir}}\right) \\
& -\frac{1}{\left|M_{A_{b}}\right| !\left|M_{A_{t}}\right| !\left(\left|R_{A_{b} 0}\right|\right)^{2}} \sum_{\Upsilon \in \Omega_{\mathrm{L}_{i, \text { not bot }}}} \operatorname{deg}\left(\operatorname{Ev}^{*} c \cap\left[\mathcal{M} \mathcal{A} \times D_{D^{r}} \mathcal{M} \mathcal{A}\right]^{\mathrm{vir}}\right)
\end{aligned}
$$

where the sum is over all quadruples $\Upsilon=\left(\Gamma_{b}, \Gamma_{t}, L, J\right) \in \Omega_{\mathrm{L}_{i, \text { not bot }}}$ as in Theorem 4.1. By choosing $c$ to be a formal sum of variables as in the definition of the correlators, we get Theorem 4.14.

\section{References}

[1] L. Caporaso and J. Harris, Counting plane curves of any genus, Invent. Math. 131(2) (1998), 345-392.

[2] Y. Eliashberg, A. Givental and H. Hofer, Introduction to symplectic field theory, Geom. Funct. Anal.(Special Volume, Part II) (2000), 560-673 GAFA 2000 (Tel Aviv, 1999).

[3] C. Faber and R. Pandharipande, Relative maps and tautological classes, J. Eur. Math. Soc. (JEMS) 7 (2005), 13-49.

[4] A. Gathmann, Absolute and relative Gromov-Witten invariants of very ample hypersurfaces, Duke Math. J. 115(2) (2002), 171-203.

[5] A. Gathmann, Gromov-Witten invariants of hypersurfaces, http://www.mathematik. uni-kl.de/gathmann/en/pub.html, 2003.

[6] E. Getzler, Intersection theory on $\bar{M}_{1,4}$ and elliptic Gromov-Witten invariants, J. Amer. Math. Soc. 10(4) (1997), 973-998.

[7] I. Goulden, D. Jackson and R. Vakil, Towards the geometry of double Hurwitz numbers, math.AG/0309440.

[8] T. Graber and R. Pandharipande, Localization of virtual classes, Invent. Math. 135(2) (1999), 487-518.

[9] T. Graber and R. Vakil, Relative virutal localization and vanishing of tautological classes on moduli spaces, Duke Math. J. 30(1) (2005), 1-37.

[10] E. Ionel and T. Parker, Relative Gromov-Witten invariants, Ann. of Math. (2), 157(1) (2003), 45-96.

[11] E. Katz, Line-bundles on stacks of relative maps, math.AG/0507322.

[12] M. Kontsevich, Enumeration of rational curves via torus actions, The moduli space of curves (Texel Island, 1994), Progress in Mathematics, 129, Birkhäuser Boston, Boston, MA, 1995, 335-368.

[13] Y.P. Lee and R. Pandharipande, A reconstruction theorem in quantum cohomology and quantum K-theory, Amer. J. Math. 126(6) (2004), 1367-1379.

[14] A.-M. Li and Y. Ruan, Symplectic surgery and Gromov-Witten invariants of CalabiYau 3-folds, Invent. Math. 145(1) (2001), 151-218.

[15] J. Li, Stable morphisms to singular schemes and relative stable morphisms, J. Differential Geom. 57(3) (2001), 509-578.

[16] J. Li, A degeneration formula of $G W$-invariants, J. Differential Geom. 60(2) (2002), 199-293. 
[17] J. Li and G. Tian, Virtual moduli cycles and Gromov-Witten invariants of algebraic varieties, J. Amer. Math. Soc. 11(1) (1998), 119-174.

[18] C. Liu, K. Liu, and J. Zhou, A formula of two-partition Hodge integrals, J. Amer. Math. Soc. 20(1) (2007), 149-184 (electronic).

[19] A. Okounkov and R. Pandharipande, Virasoro constraints for target curves, Invent. Math. 163(1) (2006), 47-108.

[20] Z. Ran, Enumerative geometry of singular plane curves, Invent. Math. 97(3) (1989), 447-465.

[21] R. Vakil, Counting curves on rational surfaces, Manuscripta Math. 102 (2000), 53-84.

[22] R. Vakil, The enumerative geometry of rational and elliptic curves in projective space, J. Reine Angew. Math. 529 (2000), 101-153.

[23] A. Vistoli. Intersection theory on algebraic stacks and on their moduli spaces, Invent. Math. 97(3) (1989), 613-670.

[24] E. Witten, Two-dimensional quantum gravity and intersection theory on moduli space, Surveys in differential geomtry, Lehigh University, Bethlehem, PA, 1991, 243-310.

Department of Mathematics

UNIVERSITY OF TEXAS

1 University Station C1200

Austin, TX 78712

E-mail address: eekatz@math.utexas.edu

Received 07/05/2006, accepted 04/10/2007. 
